\title{
Convergence Properties of Posttranslationally Modified Protein-Protein Switching Networks with Fast Decay Rates
}

\section{Authors: Gaoyang Fan, Bree Cummins, and Tomáš Gedeon}

The final publication is available at Springer via https://dx.doi.org/10.1007/s11538-016-0175-z.

Fan, G., B. Cummins, and T. Gedeon. "Convergence Properties of Posttranslationally Modified Protein-Protein Switching Networks with Fast Decay Rates." Bulletin of Mathematical Biology 78, no. 6 (June 2016): 1077-1120. 
Noname manuscript No.

(will be inserted by the editor)

\title{
Convergence properties of post-translationally modified protein-protein switching networks with fast decay rates
}

\author{
Gaoyang Fan • Bree Cummins · Tomáš \\ Gedeon
}

\begin{abstract}
A significant conceptual difficulty in the use of switching systems to model regulatory networks is the presence of so-called "black walls," codimension 1 regions of phase space with a vector field pointing inward on both sides of the hyperplane. Black walls result from the existence of direct negative self-regulation in the system. One biologically inspired way of removing black walls is the introduction of intermediate variables that mediate the negative self-regulation. In this paper, we study such a perturbation. We replace a switching system with a higher-dimensional switching system with rapidly decaying intermediate proteins, and compare the dynamics between the two systems. We find that the while the individual solutions of the original system can be approximated for a finite time by solutions of a sufficiently close perturbed system, there are always solutions that are not well approximated for any fixed perturbation. We also study a particular example, where global basins of attraction of the perturbed system have a strikingly different form than those of the original system. We perform this analysis using techniques that are adapted to dealing with non-smooth systems.
\end{abstract}

\footnotetext{
G. Fan

Department of Mathematics

University of Utah

Salt Lake City, UT 84112-0090

E-mail: gfan@math.utah.edu

B. Cummins

Department of Mathematical Sciences

Montana State University

Bozeman, MT 59717

E-mail: cummins@math.montana.edu

T. Gedeon

Department of Mathematical Sciences

Montana State University

Bozeman, MT 59717

E-mail: gedeon@math.montana.edu
} 
Keywords Gene regulation · Piecewise-linear - Singular perturbation · Transcription/translation model $\cdot$ Negative self-regulation

\section{Introduction}

One of the great challenges in systems biology is the ability to understand the emergent behavior of cellular networks that is not a direct consequence of the pathway and/or network structure. Such emergent behavior should be predictable from a proper mathematical model, but there are three fundamental challenges in constructing such models: (1) the size of the networks, (2) the low resolution of experimental measurements needed to parameterize the models, and (3) variability of functional expression in different conditions. The theory of dynamical systems has highlighted the importance and ubiquity of sensitivity to initial conditions (chaos) and sensitivity to parameter values (bifurcations) [17]. This theory, motivated by physics, is ill equipped to incorporate and interpret biological measurements where data is typically reported in terms of relative or $n$-fold expression levels. Additionally, homologous regulatory networks in different cell lines [21] or organisms lead to different functional states suggesting that system parameters play an essential role.

Current approaches to modeling gene network dynamics are characterized by a trade-off between the model's ability to quantitatively match the experimental data, and the need for a large number of kinetic parameters to parameterize the model $[8,10,13,12]$. A popular modeling platform is that of Boolean networks, where each protein, ligand or mRNA is assumed to have two states ( $\mathrm{ON}$ and $\mathrm{OFF}$ ), and the discrete time evolution of the states is based on logic-like, or Boolean update functions [1, 20, 22, 23].

The highly constrained character of the states and the update rules allows relatively easy parameterization of the model from data, however it also limits the power of generalization of the model and the quantitative match with data is typically low. Alternatively, properly parameterized ODE models can provide a good quantitative match and the power of generalization is high [24]. However, standard use and interpretation of these models requires knowledge of kinetic parameters that are usually not known. The indirect estimate of these parameters by comparing the output of the model to the experimental data suffers from at least three fundamental problems: (i) the correspondence between the dynamics and the structure of the network is not one-to-one; (ii) the need to match data corrupted by significant intrinsic and experimental noise to an individual solution of the ODE model; and (iii) the lack of methods to search high dimensional parameter spaces for dynamic signatures observed in the data.

In this situation switching networks became a popular framework to model cellular and gene regulation, since they combine discreteness of the states of Boolean networks with continuous time evolution of ODE models. The defining assumption in these models is that the effect of regulatory proteins on downstream proteins is mediated through hard thresholds; as the concentration 
of the regulator moves through a finite number of thresholds, its regulatory effects on other proteins jump discontinuously. The key advantage is that solutions of a switching system described by piecewise affine ordinary differential equations can be found explicitly while concentrations are between thresholds. If such solutions can be extended by continuity across the thresholds to all phase space, then these models are well-posed, and can be perturbed to the more commonly used Hill function models with high Hill exponents [6]. In this manner, switching networks can swiftly approximate the dynamics of smooth ODE models, permitting a more informed choice of kinematic parameters.

The central difficulty in switching models is that the existence of a variable that negatively self-regulates (i.e. there exists a negative self-loop in the graph of interactions) implies the existence of a so-called "black wall" - a subset of a co-dimension one hyperplane corresponding to a threshold, which attracts solutions from both sides. It is not a priori clear whether one can define a meaningful flow on black walls, and if so, how to analyze the $\omega$-limit sets of solutions on a black wall.

There are three principal methods that aim to resolve this problem. The first is Filippov theory $[9,15]$ that uses set-valued solutions. We will not discuss this approach in any detail in this paper. The second approach $[11,18]$ seeks to define the solutions on the black wall as limits of solutions of nearby systems, where the discontinuous nonlinearity has been smoothed out. The steepness of the nonlinearity is parameterized by a small parameter $q>0$ in such a way that $q=0$ corresponds to the switching system.

The third, more recent approach [5] uses insight from biology that negative self-regulation is rarely direct, and usually acts through intermediaries. Introduction of an intermediary variable for each negative self-regulation will remove black walls and thus will resolve the mathematically thorny issue of defining and continuing solutions on black walls. However, the question that must be asked is whether the original model and the model with added intermediary variables have similar dynamics. Clearly, in order to have a hope to resolve this question in a positive way, the system with added variables must be in some sense close to the original one. This is achieved by assuming that the dynamics of the intermediary are much faster than the dynamics of the original variable and therefore the size of the perturbation is measured by a small parameter $\epsilon$.

Our main goal in this paper is to compare and contrast the two kinds of perturbations, $\epsilon>0$ and $q>0$, described in the previous paragraphs. In the appendix of a recent paper [5], the authors consider a system where the intermediary variable is modeled as a switching variable (another option is to use a linear equation; see [5]). The original system usually represents the interaction of proteins; using motivation from cell biology, switching intermediary variables represent post-translationally modified forms of these proteins. We will call such a perturbed system a post-translationally modified protein-protein system (PTM system). The authors considered the convergence properties of a PTM system with infinitely fast decay of the modified proteins. In particular, 
they analyzed the situation when $\epsilon \rightarrow 0$ with a fixed $q>0$, and then took the limit as $q \rightarrow 0$.

We extend the analysis of [5]. We first study an $\epsilon$ perturbation with $q=0$ in a setting where we consider a solution of a switching system that enters, continues along, and then exits a black wall. We show that if we prescribe a $\delta$-neighborhood of the exit point and fix an initial condition whose trajectory encounters the black wall, one can choose $\epsilon$ sufficiently small so that the solution under the $\epsilon$ perturbation will pass within $\delta$ of the exit. However, we also show that if we fix the perturbation size $\epsilon$ first, there is a collection of interweaving regions of initial conditions that will end up outside of the $\delta$ neighborhood of the exit point. The principal reason is that the introduction of a new variable into a negative feedback loop creates a rotating and contracting vector field around what used to be the black wall; the preimage of an $\delta$ neighborhood of the exit point in this flow forms a complex structure in the phase space, and the complement of this structure will not pass close to the exit.

Since similar dynamical features (a rotating vector field) are responsible for complex global dynamics in examples like the Shilnikov attractor, in the second half of the paper we explore the possibility that the rotating vector field introduced by the new variable has consequences for the global dynamics. We study a 2D example by Plahte and Kjøglum [18] with a black wall adjacent to a white wall. Using smooth $q$-approximations we show that there are two attracting equilibria $A$ and $B$ and describe the basins of attraction. We then introduce an additional switching variable that removes the black wall, and study the correspondence between the solutions of the $2 \mathrm{D}$ problem and a $3 \mathrm{D} \epsilon$-perturbation. We show, first, that there are corresponding equilibria $\hat{A}$ and $\hat{B}$ to $A$ and $B$. Hence it makes sense to pose questions about how the basins of attraction of $A$ and $B$ correspond to those of $\hat{A}$ and $\hat{B}$ after taking the appropriate projection from $\mathbb{R}^{3}$ to $\mathbb{R}^{2}$. We find that for any $\epsilon>0$ the projection of the basin of $\hat{A}$ overlaps the interior of the basin of attraction of $B$ and therefore the global dynamics of the $3 \mathrm{D}$ system do not match the global dynamics of the 2D system for an arbitrarily small size of the perturbation $\epsilon$.

Our work illuminates the delicate nature of the perturbation where an additional switching variable is used to resolve the existence of a black wall in switching system. First, while any fixed solution can be approximated for any finite time by a solution of the enlarged system for sufficiently small $\epsilon$, this approximation does not hold uniformly for all initial data (see Section 4). Second, as we show in an example in Section 5, the perturbations of a switching system in $q$ and $\epsilon$ behave differently, in that the basins of attraction of the global attractors are distinct. We show the existence of a set of initial conditions that converge to one attractor in the $q>0$ perturbation, but their corresponding solutions in the perturbed system converge to either attractor as the size of the $\epsilon>0$ perturbation tends to zero. 


\section{Network models}

Consider a system of differential equations

$$
\dot{x}_{i}=F_{i}\left(X_{i}\right)-\gamma_{i} x_{i}, i=1, \ldots, n,
$$

that models the interaction between $n$ proteins with concentrations $x_{i}$, mediated by regulatory switches $X_{i}$. The positive constant $\gamma_{i}$ is the decay rate of protein $x_{i}$ and $F_{i}$ is a multilinear function (affine in each term) of the regulatory switches in the vector $X_{i}$. The nonlinear functions $X_{i}=\left(X_{i j}^{ \pm}\right)_{j \subset\{1, \ldots, n\}}$, where $X_{i j}$ is a function of $x_{j}$, model the switch-like effect of $x_{j}$ on $x_{i}$. The effect may be either activating or repressing, as denoted by the superscripts \pm . These interactions may be visualized through an interaction graph as shown in Figure 1, in which activation and repression are denoted by different arrows.

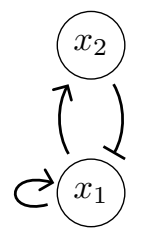

Fig. 1: An example protein interaction graph with two proteins. Sharp arrows denote positive regulation and blunt arrows denote negative regulation.

The $X_{i j}$ may either be steep sigmoids or step functions. A small parameter $q$ controls the steepness of the switch when $X_{i j}$ is a sigmoid. In this manuscript, we will say that for $q>0$, the smooth switches are Hill functions, given by

$$
\begin{gathered}
X_{i j}^{+}\left(x_{j} ; q>0\right):=\frac{x_{j}^{1 / q}}{\theta_{i j}^{1 / q}+x_{j}^{1 / q}} \\
X_{i j}^{-}\left(x_{j} ; q>0\right):=\frac{\theta_{i j}^{1 / q}}{\theta_{i j}^{1 / q}+x_{j}^{1 / q}} .
\end{gathered}
$$

The $\theta_{i j}$ are called thresholds and represent the level of $x_{j}$ required to affect $x_{i}$.

Assumption 21 Every threshold $\theta_{i j}$ of $x_{j}$ regulates one and only one $x_{i}$. This assumption is generic in the set of all choices of threshold parameters.

Assumption 21 is fundamental to our analysis of the dynamics of the system and has been used frequently in the past $[9,11,19]$. It can be convenient to assume that a transcription factor controls several downstream genes at the same threshold, in particular since there is often limited experimental evidence for what the value of the threshold may be. However, since the thresholds themselves are only abstractions of a steep, but gradual cellular responses, and since 
the experimental results will always be strongly influenced by noise, we feel that the assumption (21) is not overly restrictive. In Figure 1, Assumption 21 enforces that the two outward edges from $x_{1}$ have distinct thresholds. The third edge in the graph has no such restriction, since the variable $x_{2}$ regulates only one variable.

For $x_{j} \neq \theta_{i j}$, the limits of the Hill functions are the step functions.

$$
\begin{aligned}
& X_{i j}^{+}\left(x_{j}\right):=\lim _{q \rightarrow 0} X_{i j}^{+}\left(x_{j} ; q>0\right)=\left\{\begin{array}{l}
0, x_{j}<\theta_{i j} \\
1, x_{j}>\theta_{i j}
\end{array}\right. \\
& X_{i j}^{-}\left(x_{j}\right):=\lim _{q \rightarrow 0} X_{i j}^{-}\left(x_{j} ; q>0\right)=\left\{\begin{array}{l}
1, x_{j}<\theta_{i j} \\
0, x_{j}>\theta_{i j}
\end{array}\right.
\end{aligned}
$$

At $x_{j}=\theta_{i j}$, the $\operatorname{limit} \lim _{q \rightarrow 0} X_{i j}^{ \pm}\left(x_{j}=\theta_{i j}, q>0\right)=1 / 2$ exists. However, the value of step functions at thresholds is traditionally left undefined.

Throughout the remainder of the paper, $X_{i j}^{ \pm}$will almost always refer to the discontinuous step nonlinearity, which we also call the " $q=0$ " case. We will be clear when we discuss $q>0$ sigmoids.

Assumption 22 Consider (1) with either Hill functions or step functions $(q \geq 0)$. The coefficients of the multilinear functions $F_{i}$ in (1) are chosen such that the solutions $x(t)$ to (1) with positive initial conditions $x(0)$ remain in the positive quadrant $[0, \infty)^{n} \subset \mathbb{R}^{n}$ for all time. One possible choice is to take all coefficients positive.

\subsection{Extended system}

As we will describe in the next section, the significant advantages in ease of analysis of switching system (1) with $q=0$, compared to $q>0$ systems, is tempered by one analytical difficulty. The presence of negative self-regulation (terms like $X_{i i}^{-}$or $-X_{i i}^{+}$) introduces difficulties in defining solutions for an open set of initial conditions near, and at, some of the threshold hyperplanes. One solution to these difficulties is to introduce intermediary variables. Using biology as a motivation, the variables $x_{i}$ represent proteins and one choice is to add in an mRNA for each protein species. Then the proteins regulate the mRNA in a switch-like fashion, while the mRNA linearly controls the growth rate of the proteins [5]. Another choice is to add in intermediary proteins that have undergone a cascade of post-translational modifications before regulating other proteins. The modified proteins are assumed to have a switch-like growth rate dependent only on their progenitor protein, while the original set of proteins is now regulated by the modified proteins, also in switch-like manner [5]. Both of the choices to address negative self-regulation have their biological justification. In this paper we concentrate on the post-transcriptionally modified proteins, leaving the addition of mRNA for future work.

In general, the dynamics of such extended and original systems may not be close; however, if the modified proteins decay faster than their original versions, then singular perturbation theory gives hope that the dynamics can be 
close in the limit of sufficiently fast modified protein decay. Note however, that the classical singular perturbation theory requires smoothness and therefore is not directly applicable when $q=0$; i.e. the nonlinear switches are discontinuous. Going forward, we assume that the modified proteins decay quickly in comparison to their parent protein. The previous paragraph motivates an extension of the model (1) of the following form:

$$
\begin{aligned}
\dot{x}_{i} & =F_{i}\left(Z_{i i}^{-}, X_{i}\right)-\gamma_{i} x_{i}, i=1, \ldots n \\
\dot{z}_{i} & =\frac{1}{\epsilon}\left(A_{i}\left(X_{i i}^{+}\right)-z_{i}\right), i \in \mathbb{I} \subset\{1, \ldots n\} .
\end{aligned}
$$

In this system, I indexes the set of proteins with a negative self-regulation term. For each $i \in \mathbb{I}$ we introduce a modified version $z_{i}$ of the protein $x_{i}$ in which the self-regulation switch $X_{i i}^{-}\left(\right.$or $\left.-X_{i i}^{+}\right)$is replaced in $F_{i}$ by $Z_{i i}^{-}$(or $-Z_{i i}^{+}$). Therefore the set of switches $\tilde{X}_{i}:=X_{i} \backslash X_{i i}^{-}$contains all the original switches except the negative self-regulation switch $X_{i i}^{-}$. The switching function $Z_{i i}^{-}$is analogous to (3) and the notation reflects the dependence on the underlying variable $z_{i}$ rather than $x_{i}$. The equation for the modified protein variable $z_{i}$ is governed by an increasing affine function $A_{i}$ of $X_{i i}^{+}$, and by a small parameter $\epsilon$ controlling the decay rate.

There are several other parameters besides $\epsilon$ that can be chosen freely for the new $z_{i}$ equation: the value of the threshold at which $z_{i}$ affects $x_{i}$, value of the threshold at which $x_{i}$ affects $z_{i}$, and the coefficients of the affine function $A_{i}$. Our main effort will be chose these in such a way that the dynamics of (4) are close to that of (1) for small enough $\epsilon$ (see Remark 41).

We finish this section by noting that the extension of the network proposed in (4) which adds $|\mathbb{I}|$ variables $z_{i}, i \in \mathbb{I}$ to the original network, can be done step-by-step by adding one variable at a time. Therefore it is sufficient to compare the dynamics of a system before and after the addition of a single variable $z_{i}$, as we will do in a later section.

\section{Switching systems}

Since the nonlinearities in systems (1) and (4) with $q \geq 0$ are uniformly bounded, there exists a globally attracting compact region $\bar{\Omega} \subset[0, \infty)^{n}$ or $\bar{\Omega} \subset[0, \infty)^{n+\mathbb{I}}$ for all solutions starting in the positive orthant.

We now briefly review the main properties of the system (1) where all switches are step functions $X_{i j}^{ \pm}$with $q=0$. The main simplification of the analysis is a consequence of the fact that the thresholds divide the phase space into well defined cells.

Definition 31 Let $\theta_{i_{j}, j}<\theta_{i_{j+1}, j}$ be any two consecutive thresholds of $x_{j}$. Then a cell for system (1) has the form

$$
\kappa=\prod_{j=1}^{n}\left[\theta_{i_{j}, j}, \theta_{i_{j+1}, j}\right] \subset[0, \infty)^{n}
$$


where $\theta_{i_{j}, j}, \theta_{i_{j+1}, j}$ are either thresholds of $x_{j}$ or the lower or upper bounds of the compact region $\bar{\Omega}$.

On the interior of each cell $\kappa$, the function $F_{i}^{\kappa}:=F_{i}\left(X_{i} ; \kappa\right)$ is constant, because each component $X_{i j}^{ \pm}$attains a Boolean value, 0 or 1 . Then (1) is a decoupled linear system and can be solved exactly for $x(0) \in \kappa$ :

$$
x_{i}(t ; \kappa)=\frac{F_{i}^{\kappa}}{\gamma_{i}}+\left(x_{i}(0)-\frac{F_{i}^{\kappa}}{\gamma_{i}}\right) e^{-\gamma_{i} t}, \quad i=1, \ldots, n, \quad \text { while } x(t) \in \kappa .
$$

The solution (5) evolves toward the constant

$$
\Phi(\kappa):=\left(F_{1}^{\kappa} / \gamma_{i}, \ldots, F_{n}^{\kappa} / \gamma_{n}\right) \text { as } t \rightarrow \infty .
$$

We call $\Phi(\kappa)$ the focal point of $\kappa$. If $\Phi(\kappa) \in \operatorname{int} \kappa$, then $\Phi(\kappa)$ is a steady state of (1).

Assumption 32 No component of $\Phi(\kappa), F_{i}^{\kappa} / \gamma_{i}$, lies on a hyperplane bordering $\kappa$; i.e. $\Phi(\kappa)_{i} \neq \theta_{j i}$ for any $j$ such that $x_{i}=\theta_{j i}$ is a boundary of $\kappa$.

Definition 33 Given an $n$-dimensional cell $\kappa$, a dimension $n-1$ boundary or wall of $\kappa$ is denoted

$$
w\left(i_{k}, k\right):=\prod_{j=1}^{k-1}\left[\theta_{i_{j}, j}, \theta_{i_{j+1}, j}\right] \times\left\{\theta_{i_{k}, k}\right\} \times \prod_{j=k+1}^{n}\left[\theta_{i_{j}, j}, \theta_{i_{j+1}, j}\right]
$$

for each threshold hyperplane $x_{k}=\theta_{i_{k}, k}$ bordering $\kappa$.

The focal point $\Phi(\kappa)$ can be considered a piecewise constant function from the union of cell interiors $\bigcup$ int $(\kappa)$ to $[0, \infty)^{n}$. This function is discontinuous at a each wall $w:=\kappa \cap \bar{\kappa}$ between $\kappa$ and $\bar{\kappa}$. By Assumption 21 only one component of $\Phi(\kappa)$ and $\Phi(\bar{\kappa})$ may differ between them: $\Phi(\kappa)_{i} \neq \Phi(\bar{\kappa})_{i}$ for exactly one $i$. The wall crossings can be classified into three types.

Definition 34 Let $w(i, j)$ be a wall between two cells $\kappa$ and $\bar{\kappa}$ where $x_{j}=\theta_{i j}$. Let $\kappa$ and $\bar{\kappa}$ be named such that $x_{j}<\theta_{i j}$ in $\kappa$ and $x_{j}>\theta_{i j}$ in $\bar{\kappa}$.

1. A transparent wall occurs if $i \neq j$.

2. A white wall occurs if $i=j$ and $\Phi(\kappa)_{i}<\theta_{i i}$ and $\Phi(\bar{\kappa})_{i}>\theta_{i i}$,

3. A black wall occurs if $i=j$ and $\Phi(\kappa)_{i}>\theta_{i i}$ and $\Phi(\bar{\kappa})_{i}<\theta_{i i}$.

Along a transparent wall the solutions in each cell, $x(t ; \kappa)$ and $x(t ; \bar{\kappa})$, can be joined to form a continuous solution at a transparent wall $w(i, j)$. See Figure 9 (b) for a schematic of the flow across transparent walls and near a black wall.

A proper definition of solutions at the black and white walls presents a challenge. Since the black walls can always be reached via an open set of initial conditions, the difficulty in defining solutions along black walls poses a significant problem for the long term dynamics of the system. The most natural way to define a solution is (see [18]) 
Definition 35 The solution to system (1) with step functions $X_{i j}^{ \pm}$is defined as the limit when $q \rightarrow 0$ of the solution of (1) with Hill functions $X_{i j}^{ \pm}(q>0)$. That is, $x(t):=\lim _{q \rightarrow 0} x(t ; q>0)$.

Remark 36 In the $q=0$ case, a trajectory over multiple cells across the interiors of a finite number of transparent walls is unambiguously defined, since the flow across the interior of a transparent wall is transverse [3] to the wall. Therefore a concatenation of solutions $x(t ; \kappa), t \in[0, T]$ over multiple cells $\kappa$ is a continuous function of $t$ and for any finite $T$ it perturbs to a nearby solution of a system with $q>0$ sufficiently small.

Remark 37 As has been studied in [18], the limit as $q \rightarrow 0$ results in sliding motion along a black wall. That is, if $x_{i}=\theta_{i i}$ on a black wall when $q=0$, then the motion on the black wall is described by

$$
\dot{x}_{i}=0 ; \quad \dot{x}_{j}=F_{j}-\gamma_{j} x_{j}, \text { for } j \neq i,
$$

where $F_{j} / \gamma_{j}$ is the focal point of $x_{j}$ on both sides of the black wall.

The goal of introducing extra variables in (4) is to remove the need to consider black and white walls in (1). Since analysis of the dynamics of (1) is concerned with positively invariant sets and attractors, we will concentrate on dynamics near black walls. In order to justify this approximation, we need to compare dynamics near the black wall of (1) with the corresponding solutions of (4). We study solutions near a black wall in (1) whose forward trajectory enters the black wall $w(i, i) \subset \bar{\Omega}$. Given Assumption 32, the black wall is a result of negative self-regulation of some variable $x_{i}$ and $w(i, i) \subset\left\{x_{i}=\theta_{i i}\right\}$. We assume that the flow on $w_{i i}$ has been defined as a limit of $q \rightarrow 0$ see (7). A solution $x(t)$ either stays in the threshold hyperplane for all time, $x_{i}(t)=\theta_{i i}$ for all $t>0$, in which case it must converge to an invariant set in the hyperplane, or there is a finite time $T>0$ such that $x_{i}(t) \neq \theta_{i i}$ for $t \in(T, T+\zeta)$ for some $\zeta>0$.

Lemma 38 Let $x(0)=I^{0}$ be an initial condition on the black wall $w(i, i)$. Supposing that $x$ exits $w(i, i)$ in finite time, the exit position $x\left(T ; I^{0}\right)$ from the black wall occurs at the intersection of $w(i, i)$ with at least one other threshold hyperplane $x_{J}=\theta_{i J}$ with $J \neq i$. That is, the exit position occurs at a hyperplane where $x_{J}$ affects the variable $x_{i}$, ensuring that $x\left(T ; I^{0}\right) \in w(i, i) \cap w(i, J)$.

Proof Let $x_{i}=\theta_{i i}$ on a black wall $w(i, i)$. The flow is directed toward the black wall $w_{i . i}$ which means that $\dot{x}_{i}<0$ in the cell bordering $w_{i, i}$ with $x_{i}>\theta_{i i}$ and $\dot{x}_{i}>0$ in the cell bordering $w_{i, i}$ with $x_{i}<\theta_{i i}$. Since the direction of flow in each of these two cells is fixed, the value of $\dot{x}_{i}$ will be same until some variable $x_{J}$ that is on the right hand side of the equation of $\dot{x}_{i}$ crosses a threshold $\theta_{i J}$. Thus $x_{i}$ cannot leave the threshold hyperplane $x_{i}=\theta_{i i}$ until such a crossing.

Definition 39 An exit hyperplane of a black wall $w(i, i)$ is a hyperplane $x_{J}=$ $\theta_{i J}$ such that for a relatively open subset of initial conditions $\left\{x(0)=I^{0}\right\} \subset$ $w(i, i)$, the exit positions $\left\{x\left(T ; I^{0}\right)\right\} \subset w(i, i) \cap w(i, J)$. 
Using Definition 39 each black wall $w(i, i)$ can be divided into open regions, each of which is associated to a different exit hyperplane. The dividing surfaces between the open regions consist of initial conditions that map to the intersection of two exit hyperplanes.

\section{Local Dynamics}

As mentioned previously, a protein-only system can be augmented by multiple variables representing post-transcriptionally modified proteins, and these may be introduced one at a time. In this section, we study a system with only one additional modified protein variable in a local setting very close to a black wall. Our main result is Theorem 43 which states that there is a region in phase space arbitrarily close to a black wall where the trajectories of the protein-only system (1) and the PTM system (4) diverge. The proof is in the Appendix, where we show that the region has a complex structure, so that the regions of divergence are not easily predicted.

To facilitate comparison between the dynamics of (1) and (4) near a black wall when $q=0$, we introduce a modified notation using the following variables:

- $x$, a negatively self-regulating protein at the black wall of interest;

$-z$, a modified protein that eliminates the negative self-regulation in $x$; and

$-y_{i}$, all other variables. Note that these variables could be self-regulating at other walls.

The variables $x$ and $z$ do not have subscripts, and so we write $\theta_{x}, \gamma_{x}$, etc., in lieu of the subscript notation that we used earlier. To distinguish between the protein-only and PTM systems, we use hatted variables in the PTM equations.

For the protein-only system (1), we write

$$
\begin{aligned}
\dot{x} & =-\gamma_{x} x+F\left(X^{-}, Y_{x}\right) \\
\dot{y}_{i} & =-\gamma_{i} y_{i}+F_{i}\left(X_{i}, Y_{i}\right), i=1 \ldots n-1,
\end{aligned}
$$

where $x=\theta_{x}$ on the black wall $w(x, x)$ (i.e. $x$ is the negative self-regulation variable and $\theta_{x}$ is the black wall threshold). On the right hand side of the equations for $\dot{y}_{i}$ we single out the dependence of $y_{i}$ on the variable $x$ as step function $X_{i}$ that can take the form $X^{+}$or $X^{-}$whenever $y_{i}$ depends on $x$. Each $X_{i}$ has a distinct threshold due to Assumption 21. The vectors $Y_{x}$ and $Y_{i}$ contain step functions depending on a subset of variables $\left\{y_{j}\right\}$ regulating $x$ or $y_{i}$, respectively.

In the PTM system (4), we will add exactly one modified protein, the one that corresponds to the black wall of interest, $w(x, x)$ in the protein-only system (8). We write (4) as

$$
\begin{aligned}
\dot{\hat{x}} & =-\gamma_{x} \hat{x}+F\left(\hat{Z}^{-}, \hat{Y}_{x}\right) \\
\dot{\hat{z}} & =\frac{1}{\epsilon}\left(-\hat{z}+\left\{\begin{array}{c}
\theta_{z}+\beta_{z}, \hat{x}>\theta_{x} \\
\theta_{z}-\beta_{z}, \hat{x}<\theta_{x}
\end{array}\right)\right. \\
\dot{\hat{y}}_{i} & =-\gamma_{i} \hat{y}_{i}+F_{i}\left(\hat{X}_{i}, \hat{Y}_{i}\right) ; i=1 \ldots n-1
\end{aligned}
$$


We will take the (single) threshold of $\hat{z}$ to be $\theta_{z}$, and $\hat{x}$ is assumed to regulate $\hat{z}$ at the original value $\theta_{x}$.

Remark 41 All thresholds, decay rates, and multilinear functions $F_{i}$ are taken to be the same between systems (8) and (9).

The only free parameters are $\epsilon>0$, the threshold $\theta_{z}$, and the regulation constant $\beta_{z}$. We choose $\beta_{z}>0$ to be consistent with $\hat{x}$ up-regulating $\hat{z}$, and we choose $\theta_{z}-\beta_{z}>0$ to keep the trajectory $\hat{z}(t)>0$ for a positive initial condition. There are no further requirements on the constants $\theta_{z}$ and $\beta_{z}$. We will consider $\epsilon \ll 1$ to be a small parameter.

Throughout the work to come, we will use the following results. Let $v$ be a variable $x, y_{i}$, or $z$ in one of the systems of interest, (8) or (9). Recall Equation (5) for the flow within a cell $\kappa$, which we rewrite here for convenience:

$$
v(t)=\Phi_{v}(\kappa)+\left(v(0)-\Phi_{v}(\kappa)\right) e^{-\gamma_{v} t},
$$

remembering that $\Phi_{v}(\kappa)=F_{v}^{\kappa} / \gamma_{v}$ is the focal point for cell $\kappa$. Naturally, this equation changes between cells in the phase space. If we want to know the time from $v(0) \in \kappa$ to some location $v\left(T_{v}\right) \in \kappa$, we may solve for time in (10):

$$
T_{v}=-\frac{1}{\gamma_{v}} \ln \left(\frac{v\left(T_{v}\right)-\Phi_{v}(\kappa)}{v(0)-\Phi_{v}(\kappa)}\right) .
$$

We show in the proof of Lemma 42 in the Appendix that there is a region of interest surrounding the black wall where the $y_{i}$ and $\hat{y}_{i}$ trajectories are identical. Therefore, we assess the closeness of the protein-only and PTM solutions via the $x$ and $\hat{x}$ components of the trajectory in the vicinity of a black wall, given by (10). In order to do so we first carefully define the neighborhood of the black wall where this comparison will take place.

Recall that an exit set $E \subset A$ for a compact region $A$ with a flow $\psi(x, t)$ has the property that if $\psi(x, 0) \in A$ and $\psi(x, T) \notin A$ for some $T>0$ then there must exist $t \in[0, T)$ such that $\psi(x, t) \in E$.

\section{Lemma 42}

(I) Given a black wall $w(x, x)$ in the protein-only system, there exists a compact rectangular region $\mathcal{R} \subset[0, \infty)^{n}$ of the form

$$
\mathcal{R}:=\mathcal{R}_{x} \times \prod_{i=1}^{n-1} \mathcal{R}_{y_{i}}
$$

with $\mathcal{R} \cap w(x, x) \neq \emptyset$, which has an exit set $E \subset[0, \infty)^{n-1}$ of the form

$$
E=\left\{\left(x, y_{i}\right) \in \mathcal{R} \mid y_{J}=\theta_{x, J} \text { for a single index } J \in\{1, \ldots, n-1\}\right\}
$$

(II) Given $\mathcal{R}$, there exist

(a) a compact rectangular region $\hat{\mathcal{R}} \subset[0, \infty)^{n+1}$ of the form

$$
\hat{\mathcal{R}}:=\mathcal{R} \times\left[\theta_{z}-\beta_{z}, \theta_{z}+\beta_{z}\right]
$$


(b) an exit set $\hat{E} \subset[0, \infty)^{n}$

$\hat{E}:=\left\{\left(\hat{x}, \hat{y}_{i}, \hat{z}\right) \in \hat{\mathcal{R}} \mid \hat{y}_{J}=\theta_{x, J}\right.$ for the same index $\left.J \in\{1, \ldots, n-1\}\right\}$ as in (12)

(c) and a family of regions $\hat{\mathcal{R}}(\epsilon) \subset \hat{\mathcal{R}}$

$$
\hat{\mathcal{R}}(\epsilon)=\hat{\mathcal{R}}_{x}(\epsilon) \times\left[\theta_{z}-\beta_{z}, \theta_{z}+\beta_{z}\right] \times \prod_{i=1}^{n-1} \mathcal{R}_{y_{i}}
$$

where $\hat{\mathcal{R}}_{x}(\epsilon) \subset \mathcal{R}_{x}$ is parameterized by $\epsilon$ in $(9)$.

The sets $\hat{\mathcal{R}}, \hat{E}$ and $\hat{\mathcal{R}}(\epsilon)$ have the following two properties.

(1) For all initial conditions $\hat{I}^{0} \in \hat{\mathcal{R}}(\epsilon)$, the trajectory $\left(\hat{x}(t), \hat{y}_{i}(t), \hat{z}(t)\right)$ of (9) remains in $\hat{\mathcal{R}}$ until the exit through $\hat{E}$.

(2) For an initial condition $\hat{I}^{0} \in \hat{\mathcal{R}}(\epsilon)$ with $\hat{y}_{i}(0)=y_{i}(0)$, we have that $\hat{y}_{i}(t)=y_{i}(t)$ as long as $y_{i}(t) \in \mathcal{R}_{y_{i}}$.

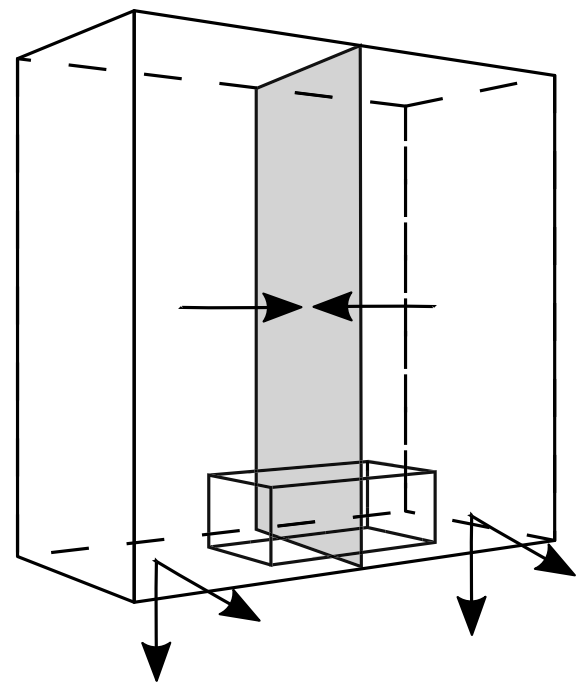

Fig. 2: A schematic of $\mathcal{R}$ intersecting a black wall in a three dimensional phase space. The arrows indicate the direction of flow of the vector field. The region consists of two cuboid cells that share a black wall (gray rectangle) as a boundary. Each of the cells has two exit hyperplanes, the bottom and front faces of the cuboids. The small cuboid intersecting the black wall is a region $\mathcal{R}$ such that trajectories within $\mathcal{R}$ will exit through the bottom hyperplane. Notice that the region $\mathcal{R}$ must be sufficiently far from the front face, since it is also an exit hyperplane.

The region $\mathcal{R}$ is a compact rectangle consisting of points near a black wall that will all eventually leave $\mathcal{R}$ through the same exit hyperplane $y_{J}=\theta_{x, J}$ 
of $w(x, x)$; see Figure 2 for an example. The region $\hat{\mathcal{R}}(\epsilon)$ is one dimension higher. To construct $\hat{\mathcal{R}}(\epsilon)$ we first take a product of $\mathcal{R}$ with an interval $\left[\theta_{z}-\right.$ $\left.\beta_{z}, \theta_{z}+\beta_{z}\right]$ in the $z$-direction and then show that we can select the $x$-component $\hat{\mathcal{R}}_{x}(\epsilon) \subset \mathcal{R}_{x}$ small enough so that solutions oscillating in the $x-z$ plane do not leave larger box $\mathcal{R} \times\left[\theta_{z}-\beta_{z}, \theta_{z}+\beta_{z}\right]$ before exiting through $\hat{E}$. The proof of Lemma 42 can be found in the Appendix.

Having defined sets $\mathcal{R}$ and $\hat{\mathcal{R}}(\epsilon)$ where we can compare the $x$ and $\hat{x}$ components of the solutions of the protein-only system (8) and the PTM system (9), we will now prove our main result. We show that for any fixed decay parameter $\epsilon>0$ and any sufficiently small $\delta$, there are initial conditions where $x$ and $\hat{x}$ differ by at least $\delta$ at the time when the trajectory of (1) exits the black wall. This is true even though for any initial condition and any $\delta>0$, one can choose an $\epsilon$ sufficiently small so that $x$ and $\hat{x}$ are closer than $\delta$ at the time of exit. In other words, the order of the quantifiers matters.

Theorem 43 Given $\mathcal{R}$ and $\hat{\mathcal{R}}(\epsilon)$, consider initial conditions $I^{0}:=\left(x(0), y_{i}(0)\right) \in$ $\mathcal{R}$ for (8) and $\hat{I}^{0}:=\left(I^{0}, \hat{z}(0)\right) \in \hat{\mathcal{R}}(\epsilon)$ for (9). Let $T_{y}$ be the travel time of the solution of (8) between the starting point $I^{0}$ and the exit hyperplane $y_{J}=\theta_{x, J}$, as calculated from (11). Then,

1. Given $\delta>0$, there exists $\epsilon\left(\delta, \hat{I}^{0}\right)$ such that $\left|x\left(T_{y}\right)-\hat{x}\left(T_{y}\right)\right| \leq \delta$.

2. Given $\epsilon>0$, there exists $\Delta(\epsilon)>0$ such that for any $0<\delta<\Delta(\epsilon)$ there exists a region with nonempty interior

$$
\begin{aligned}
& \mathcal{E}(\delta, \epsilon)=\tilde{\mathcal{E}}(\delta, \epsilon) \times \prod_{i \neq J} \mathcal{R}_{y_{i}} \subset \hat{\mathcal{R}}(\epsilon) \\
& \tilde{\mathcal{E}}(\delta, \epsilon) \subset \hat{\mathcal{R}}_{x}(\epsilon) \times\left[\theta_{z}-\beta_{z}, \theta_{z}+\beta_{z}\right] \times \mathcal{R}_{y_{J}},
\end{aligned}
$$

such that $\left|x\left(T_{y}\right)-\hat{x}\left(T_{y}\right)\right|>\delta$ for initial conditions $\hat{I}^{0} \in \mathcal{E}(\delta, \epsilon)$.

When there are initial conditions in $\mathcal{R}$ and $\hat{\mathcal{R}}(\epsilon)$ that are equal in all but the $\hat{z}$ variable, the $x$ and $\hat{x}$ components of their trajectories can be made arbitrarily close via a choice of $\epsilon$ while they stay in $\mathcal{R}$ and $\mathcal{R} \times\left[\theta_{z}-\beta_{z}, \theta_{z}+\beta_{z}\right]$ respectively. However, for any fixed $\epsilon$, there are regions of initial conditions, even close to the exit hyperplane, where the trajectories cannot be made arbitrarily close. The Theorem 43 is proven in the Appendix, where we also show that this excluded region $\mathcal{E}(\delta, \epsilon)$ has a complex structure. In the remainder of this section, we summarize the main idea behind the proof.

Consider a solution trajectory of the protein-only system that intersects the black wall. The protein-only system trajectory determines a time to the exit set $y_{J}=\theta_{x, J}$, which we call $T_{y}$. Since this trajectory intersects the black wall, at the time of exit, the $x$-variable attains value $x=\theta_{x}$. Now consider solution for the PTM system with some small fixed $\epsilon$ and the initial condition with $\hat{x}(0)=x(0), \hat{y}_{i}(0)=y_{i}(0)$ and some $\hat{z}(0)$. By Lemma $42, T_{y}$ is also the time at which the variable $\hat{y}_{J}$ attains the threshold $\theta_{x, J}$ in the PTM solution trajectory. If $\hat{x}$ is within a $\delta$ neighborhood of $\theta_{x}$ at time $T_{y}$, then we say the trajectories are close, otherwise they are far apart. The set of initial conditions where the solutions are far apart is the excluded region $\mathcal{E}(\delta, \epsilon)$. 

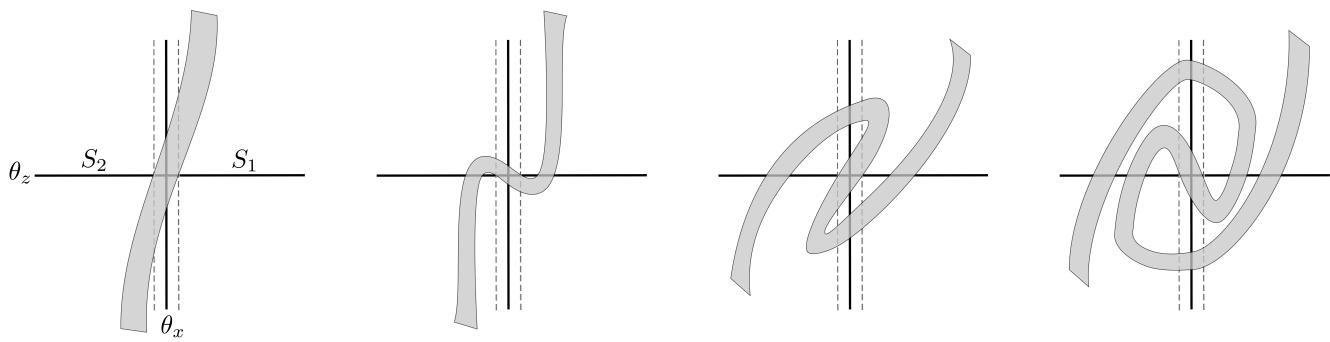

increasing $T_{y}$

Fig. 3: Conceptual picture of the preimage of the $\delta$-strip about $\theta_{x}$ in the $(\hat{x}, \hat{z})$ plane for increasing exit time $T_{y}$. The $\delta$-strip is enclosed between the dashed lines. The preimages are the shaded regions, which become more twisted as $T_{y}$ increases.

The introduction of the new variable $\hat{z}$ induces oscillations in the $\hat{x}, \hat{z}$ projection of the solution, as this solution monotonically increases or decreases along the $y_{J}$ variable. This occurs because the $\hat{x}$ and $\hat{z}$ variables form a negative feedback loop. The oscillations can carry $\hat{x}$ outside of a $\delta$ neighborhood of the plane $\hat{x}=\theta_{x}$ at time $T_{y}$. We can examine the preimage $P\left(\delta, T_{y}\right)$ in the PTM flow of the $\delta$-neighborhood $T_{y}$ time units in the past, which is a region of initial conditions satisfying $\left|\theta_{x}-\hat{x}\left(T_{y}\right)\right|<\delta$. The $\hat{x}-\hat{z}$ projection of several such preimages $P\left(\delta, T_{y}\right)$ is shown in Figure 3. Due to the oscillatory nature of $\hat{x}$ and $\hat{z}$, the preimages twist around the point $\left(\theta_{x}, \theta_{z}\right)$ more as $T_{y}$ increases.

The excluded region $\mathcal{E}(\delta, \epsilon)$ is the complement of $\bigcup_{T_{y}} P\left(\delta, T_{y}\right)$ restricted to $\hat{R}(\epsilon)$. This is clearly a region with a complicated braided structure. In the Appendix, we show that it contains a subset with $n+1$-dimensional interior for any sufficiently small $\delta$ given a fixed $\epsilon$.

The twisted structure of the excluded region $\mathcal{E}(\delta, \epsilon)$ suggests that it may be difficult to predict whether solutions to the PTM system (9) will approximate the solutions to the protein-only system (8) near a black wall or not. This leads to the question of whether the oscillations in $\hat{x}$ and $\hat{z}$ can lead to different long term dynamics (different attractors) for initial conditions near a black wall of a protein-only system. In the following section, we will explore a specific example in which oscillations in the $\hat{x}$ and $\hat{z}$ variables result in striated regions of initial conditions that lead to distinct asymptotic outcomes. The main feature of this example is the transition from a black wall to a white wall in the protein-only system. Our primary method of analysis is to look at preimages of walls in the PTM system. These preimages are not the excluded region $\mathcal{E}(\delta, \epsilon)$ discussed in this section. There is no explicit dependence on a small parameter $\delta$ in the comparison between the protein-only and PTM systems; instead the preimages are classified according to their asymptotic dynamics. 
Nonetheless, the phenomenon seen in the following section is also due to the oscillations in $\hat{x}$ and $\hat{z}$ that give rise to the preimages in Figure 3.

\section{Global Dynamics}

In this section, we will break away from local dynamics and explore the consequences of an $\epsilon>0$ modification to the global dynamics of a system. We will study the following example $[18,25]$ :

$$
\begin{aligned}
& \dot{x}=k_{x}\left(X_{x x}^{+}+Y_{x y}^{+}-2 X_{x x}^{+} Y_{x y}^{+}\right)-\gamma_{x} x \\
& \dot{y}=k_{y}\left(1-X_{y x}^{+} Y_{y y}^{+}\right)-\gamma_{y} y
\end{aligned}
$$

We choose the following parameter values: $\theta_{x x}=\theta_{y y}=0.5, \theta_{x y}=\theta_{y x}=1$, $k_{x}=k_{y}=1$, and $\gamma_{x}=0.6, \gamma_{y}=1.1$.

Since $x$ and $y$ have two thresholds each, these divide $\mathbb{R}^{2}$ into 9 cells (recall Definition 31), which will be denoted by $\kappa_{i}, i=1, \ldots, 9$, as pictured in Figure 4. We use Definition 34 to identify the type of every wall in the $q=0$ case. Note the presence of black, white, and transparent walls in Figure 4.

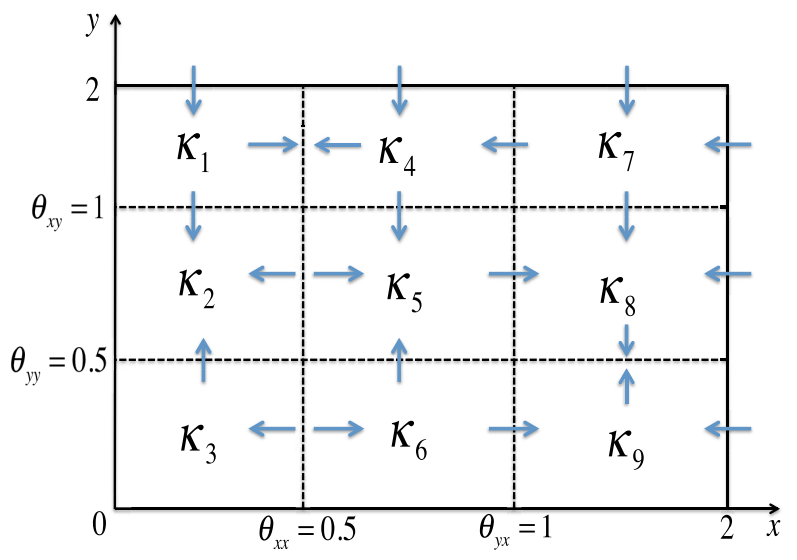

Fig. 4: The division of phase space for the system (13) with $q=0$. Equation (6) and Definition 34 are used to determine the direction of the arrows. This closed region is part of the globally attracting compact region $\bar{\Omega}$ mentioned in Section 3 .

To calculate the direction of flow indicated by the arrows in Figure 4, we find the focal points $\Phi\left(\kappa_{i}\right), i=1, \ldots, 9$ from the formula in $(6) . \Phi\left(\kappa_{i}\right)$ determines the direction of the flow out of $\kappa_{i}$. For example, the focal point of $\kappa_{1}$ is $\left(1 / \gamma_{x}, 1 / \gamma_{y}\right) \approx(1.67,0.91)$. Therefore any trajectory beginning in $\kappa_{1}$ is increasing in $x$ and decreasing in $y$, hence the two arrows departing from the cell $\kappa_{1}$. 
The nine cells pictured in Figure 4 form a globally attracting compact region $\bar{\Omega} \subset[0, \infty)^{2}$ as introduced in Section 3. To see this, consider the upper half-plane $y>2$. In this half-plane a straightforward estimate in (13) shows that $\dot{y}<1-2(1.1)<0$. A similar calculation exists for the half-plane $x>2$ and so every initial condition outside the nine-cell region in Figure 4 gives rise to a trajectory that enters the region, where the nine-cell region is considered to be a subset of $[0, \infty)^{2}$. We remark that the flow does not exist across the left and lower boundaries, $x=0$ and $y=0$, since the flow is undefined on the far side. Because of this topology, the line segments $\{x=0\} \times[0,2]$ and $[0,2] \times\{y=0\}$ are in the interior of the nine-cell region. Along these line segments, it can be verified that the flow is either tangential toward a fixed point or pointing inward.

As we remarked in Section 3, when a focal point of cell $\Phi(\kappa)$ is in int $\kappa$, then $\Phi(\kappa)$ is an attracting equilibrium of the system. From Figure 4 and this remark, we can see that there is an attracting equilibrium of the system in $\kappa_{2}$. This steady state is given by

$$
A:=\left(0,1 / \gamma_{y}\right)
$$

There are no other attracting equilibria in the interiors of $\kappa_{i}$, as can be confirmed by examining Figure 4. However there may be equilibria on the boundaries of the cells. Numerical evidence suggests that for $q>0$ there is a saddle point in $\kappa_{2}$ that converges toward the white wall between $\kappa_{2}$ and $\kappa_{5}$ as $q \rightarrow 0$, and a steady state in $\kappa_{9}$ that converges upward toward the black wall between $\kappa_{8}$ and $\kappa_{9}$. Proving the existence and type of these equilibria is beyond the scope of this paper. For rigorous work exploring equilibria on walls, see $[5,11,16,18,19,25]$ for approaches using singular perturbation theory and see $[2,9,15]$ for approaches using Filippov theory. For our purposes, it sufficient to note the following.

Lemma 51 For $q=0$, the region $\kappa_{5} \cup \kappa_{6} \cup \kappa_{8} \cup \kappa_{9}$ is a trapping region (in other words, a positively invariant region) that we denote $B$ in system (13).

Proof This follows immediately from examination of the vector field.

We will use the fact that any initial condition in the interior of the cell $\kappa_{5}$ remains in $B$ and does not approach the steady state $A$.

The true complexity of example (13) occurs at the intersection point $\mathcal{X}:=$ $\left(\theta_{x x}, \theta_{x y}\right)$, which marks the transition from a black wall $w(x, x)$ between $\kappa_{1}$ and $\kappa_{4}$ to a white wall between $\kappa_{2}$ and $\kappa_{5}$. There is no unique solution for the initial condition $\mathcal{X}$ when $q=0$; there is instead a cone of potential solutions fanning out from $\mathcal{X}$ [9]. Since an open set of solutions in $\kappa_{1} \cup \kappa_{4}$ enters the black wall and slides down towards $\mathcal{X}$, resolution of forward trajectory from $\mathcal{X}$ is very important. We will consider the open region of initial conditions in $\kappa_{1}$ with trajectories that intersect the black wall. In order to determine forward trajectories in cell $\kappa_{1}$ we examine the solution to (13) when $q>0$, and then take a limit as $q \rightarrow 0[11,18]$. We start with the following observation of the flow direction along the line segment $\left\{\theta_{x x}\right\} \times\left(1 / \gamma_{y}, 2\right]$ when $q>0$. 
Theorem 52 For all $q>0, \dot{x}>0$ and $\dot{y}<0$ on $\left\{\theta_{x x}\right\} \times\left(1 / \gamma_{y}, 2\right]$ uniformly in $q$.

Proof Recall that

$$
\dot{x}=X_{x x}^{+}+Y_{x y}^{+}-2 X_{x x}^{+} Y_{x y}^{+}-0.6 x .
$$

Referring back to the equation for a Hill function in (2), we see that $X_{x x}^{+}=0.5$ when $x=\theta_{x x}=0.5$. Then for any $y>0$ we have

$$
\left.\dot{x}\right|_{\theta_{x x}}=0.5-0.6(0.5)>0 \text {. }
$$

Likewise,

$$
\dot{y}=1-X_{y x}^{+} Y_{y y}^{+}-1.1 y
$$

which leads to

$$
\dot{y} \leq 1-1.1 y<0 \text { for } y \in\left(1 / \gamma_{y}, 2\right] .
$$

It is clear that these bounds are independent of $q$, and therefore neither derivative approaches 0 as $q \rightarrow 0$.

Corollary 53 In the limit as $q \rightarrow 0$, any trajectory intersecting the point $\left(\theta_{x x}, \theta_{x y}\right)$ enters the trapping region $B$.

Proof First note that $\left(\theta_{x x}, \theta_{x y}\right) \in\left\{\theta_{x x}\right\} \times\left(1 / \gamma_{y}, 2\right]$. From the proof of Theorem 52 , we know that $\dot{x}$ evaluated at the intersection point $\left(\theta_{x x}, \theta_{x y}\right)$ is constant, and $\dot{y}$ at the same point has the form

$$
\left.\dot{y}\right|_{\left(\theta_{x x}, \theta_{x y}\right)}=-0.1-\frac{0.5^{1 / q}}{\left(1+0.5^{1 / q}\right)^{2}}
$$

from (15). As $q \rightarrow 0, \dot{y}$ approaches the value -0.1 from below monotonically. Thus any limit $q \rightarrow 0$ of trajectories of (13) with $q>0$ that passes through $\left(\theta_{x x}, \theta_{x y}\right)$ has a forward path in the direction of $(\dot{x}>0, \dot{y}<0)$ from Theorem 52 , and enters $\kappa_{5}$.

We now examine the global dynamics for initial conditions in the cell $\kappa_{1}$.

Theorem 54 In system (13) with $q=0$, there exists an separatrix in the cell $\kappa_{1}$ where an initial condition starting above the separatrix goes to the trapping region $B$ in Lemma 51 and an initial condition starting below the separatrix goes to equilibrium $A$ defined in (14).

Proof For every initial condition $(x(0), y(0)) \in \operatorname{int}\left(\kappa_{1}\right)$, there is a unique forward solution within $\kappa_{1}$. For any initial condition, one can calculate the time $T_{y}$ needed to reach $\left\{y=\theta_{x y}\right\}$ and the time $T_{x}$ needed to reach $\left\{x=\theta_{x x}\right\}$ using the formula in (11). We list these calculations here for clarity, using the fact that the focal points are known, $\Phi_{x}\left(\kappa_{1}\right)=1 / \gamma_{x}$ and $\Phi_{y}\left(\kappa_{1}\right)=1 / \gamma_{y}$ :

$$
T_{x}=\frac{1}{\gamma_{x}} \ln \frac{x(0)-1 / \gamma_{x}}{\theta_{x x}-1 / \gamma_{x}} ; \quad T_{y}=\frac{1}{\gamma_{y}} \ln \frac{y(0)-1 / \gamma_{y}}{\theta_{x y}-1 / \gamma_{y}} .
$$


Then $C:=\left\{(x(0), y(0)) \mid T_{x}=T_{y}\right\}$ is the curve in $\kappa_{1}$ that separates initial conditions that converge to $A$ and $B$. To show this, we solve $T_{x}=T_{y}$ to derive the expression for the curve:

$$
C(x(0)):=y(0)=1 / \gamma_{y}+\left(\theta_{x y}-1 / \gamma_{y}\right)\left(\frac{1 / \gamma_{x}-x(0)}{1 / \gamma_{x}-\theta_{x x}}\right)^{\gamma_{y} / \gamma_{x}}
$$

The expression on the right hand side is well-defined, since $x(0) \leq \theta_{x x}<1 / \gamma_{x}$. By the same reason, the fraction in (16) is greater than 1 , so that $y(0) \geq \theta_{x y}$. The cell $\kappa_{1}$ lies in this region, $\kappa_{1} \subset\left\{x(0) \leq \theta_{x x}\right\} \times\left\{y(0) \geq \theta_{x y}\right\}$. Therefore $C$ is a well-defined 1-dimensional curve in $\kappa_{1}$ given by (16) that partitions the interior of $\kappa_{1}$ into two regions.

Choose an initial condition above the separatrix; i.e., take $(x(0), y(0))$ such that $y(0)>C(x(0))$. Then, $T_{y}>T_{x}$, and the trajectory will reach $\left\{x=\theta_{x x}\right\}$ before $\left\{y=\theta_{x y}\right\}$. In other words, it intersects the black wall at some point with $y\left(T_{x}\right)>\theta_{x y}$. By Remark 37, there is sliding motion along the wall. By Theorem $52, \dot{y}<0$ for every $y \geq \theta_{x y}$ uniformly in $q$, and so the finite time sliding motion is downward toward the point $\left(\theta_{x x}, \theta_{x y}\right)$. Then by Corollary 53 , this trajectory will enter the trapping region $B$.

Likewise, choosing $(x(0), y(0))$ such that $y(0)<C(x(0))$ implies $T_{y}<T_{x}$. Such a trajectory enters $\kappa_{2}$ instead of reaching the black wall, and hence travels toward equilibrium $A$.

\section{$5.13 \mathrm{D}$ system}

We now add an additional $\hat{z}$ variable to the $q=0$ system (13) to resolve the negative self-regulation in the $x$ variable. We can safely ignore the negative self-regulation in the $y$ variable, since the corresponding black wall lies outside of the region of interest. The new three dimensional system is governed by the following equations:

$$
\begin{aligned}
\dot{\hat{x}} & =k_{x}\left(Z_{x z}^{+}+Y_{x y}^{+}-2 Z_{x z}^{+} Y_{x y}^{+}\right)-\gamma_{x} \hat{x} \\
\dot{\hat{z}} & =\epsilon^{-1}\left(-\hat{z}+k_{z} X_{z x}^{+}\right) \\
\dot{y} & =k_{y}\left(1-X_{y x}^{+} Y_{y y}^{+}\right)-\gamma_{y} y
\end{aligned}
$$

We will use the same parameters as before, along with $\theta_{x z}=0.5, \epsilon=0.5$ and $k_{z}=1$. Notice that $\theta_{z x}=\theta_{x x}$ to match the previous parameters.

\subsection{State Transition Diagram for the 3D system}

We wish to emphasize the locations of the walls in the 3D system, which is not easily done in traditional phase space. We instead use a state transition diagram, as introduced in [7]. The diagram represents each cell in the phase space as a vertex. Each wall between two cells is represented as an edge between 
the corresponding vertices. Within each cell the flow is affine and focused toward the target point. In our example, there are three variables, $\hat{x}, \hat{y}$ and $\hat{z}$, in which variables $\hat{x}$ and $\hat{y}$ have two thresholds each and $\hat{z}$ has one. This leads to a partitioning of phase space into 18 cells. Solving for target points in each cell we arrive at state transition diagram in Figure 5.

The flow results in a well defined direction on an edge if it corresponds to a transparent wall. Since the black walls $\left(y=\theta_{y y}, \hat{x}>\theta_{y x}, \hat{z}>\theta_{x z}\right)$ and $\left(y=\theta_{y y}, \hat{x}>\theta_{y x}, \hat{z}<\theta_{x z}\right)$ are approached by the flow in both co-cells, we represent this by two arrows pointing toward each other along the edge that represents that wall. White walls would have two arrows pointing away from each other along the edge, but there are none in the $3 \mathrm{D}$ system (17). The state transition diagram represents gross features of the flow. In particular, no solution of the system (17) can transition between the states in a direction that does not respect the arrows. However, it is not true that each path in the state transition graph must be realizable by a solution of (17).

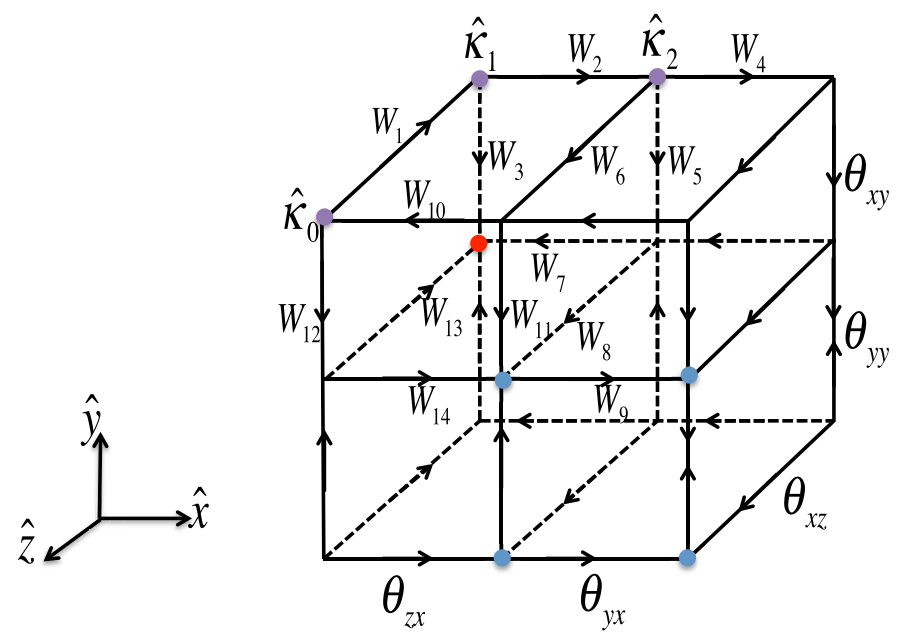

Fig. 5: The state transition diagram for the system in (17), with the axes on the left indicating the directions of increase for $\hat{x}, \hat{y}$, and $\hat{z}$. Each directed edge represents a wall between two cells, with a threshold value as indicated by $\theta_{y x}, \theta_{z x}, \theta_{x z}, \theta_{x y}$, and $\theta_{y y}$ on the bottom and right hand side of the figure. The directions along each edge are determined by the focal points of the system within each cell, where the cells are the vertices of the diagram. The cells $\hat{\kappa}_{0}, \hat{\kappa}_{1}$, and $\hat{\kappa}_{2}$ are used in the text to describe some of the walls $W_{i}, i=1, \ldots, 14$ (see Figure 6 for more separated wall labels). The red cell contains a steady state of the system analogous to the steady state $A$ in (14) and the blue cells are a trapping region analogous to $B$ described in Lemma 51 . 
5.3 Attractors in the $3 \mathrm{D}$ system

We now analyze the state transition diagram. Note that there are significant similarities and differences between 3D state transition diagram in Figure 5 and $2 \mathrm{D}$ state transition diagram in Figure 4 . The first observation is that the presence of the $\hat{z}$ variable doubled the number of cells. However, the $y$ variable dynamics is the same between the two systems and so across the upper $y$ threshold the flow has $\dot{y}<0$; the flow across the lower threshold is also analogous. The most important difference is that the black wall between $\kappa_{1}$ and $\kappa_{4}$ and the white wall between $\kappa_{2}$ and $\kappa_{5}$ disappears.

Analyzing Figure 5 we observe that there is one cell $\left[0, \theta_{z x}\right] \times\left[0, \theta_{x z}\right] \times$ $\left[\theta_{y y}, \theta_{x y}\right]$, corresponding to the state denoted by a red dot, where all of the surrounding walls are transparent and flow is going in. This indicates that this cell contains an attracting equilibrium. To verify this, we solve the equations $(17)$ in the cell $\left[0, \theta_{z x}\right] \times\left[0, \theta_{x z}\right] \times\left[\theta_{y y}, \theta_{x y}\right]$

$$
\dot{\hat{x}}=-\gamma_{x} \hat{x}, \quad \dot{\hat{z}}=-\epsilon^{-1} \hat{z} ; \quad \dot{y}=-\gamma_{y} y+1 .
$$

for an equilibrium. The result $\hat{A}=\left(0,0,1 / \gamma_{y}\right)$ lies itself in this cell, and so it is an attracting equilibrium for the flow. Note that our notation reflects that this point is a direct analog of the point $A$ in the 2D flow in (14); the only difference is that the new variable $\hat{z}=0$ in $3 \mathrm{D}$ case.

On the other hand, we observe from Figure 5 that the four blue cells on the bottom front of the state transition diagram are positively invariant. Therefore this collection of cells contains an attractor. The proof that this attractor is actually an equilibrium on a black wall is beyond the scope of this paper. We call this trapping region $\hat{B}$, because it is a direct analog of the $2 \mathrm{D}$ trapping region $B$ from Lemma 51.

To summarize this section, the dynamics of both systems (13) and (17) for $q=0$ have two attractors: an equilibrium $A, \hat{A}$ and another attractor in a trapping region $B, \hat{B}$. The basins of attraction of these two attractors in $2 \mathrm{D}$ system (13) are simple: Theorem 54 computes the separatrix between these global attractors in $\kappa_{1}$. In contrast, as we will see next, the basins of attraction of $A$ and $B$ in $3 \mathrm{D}$ system (17) are much more complex, and intricately interwoven.

\subsection{Structure of the basins of attraction}

In the $2 \mathrm{D}$ system (13) we studied the basins of attraction of $A$ and $B$ in the cell $\kappa_{1}$ and found a single separatrix between them in Theorem 54 . In the 3D system, $\kappa_{1}$ corresponds to two cells $\hat{\kappa}_{0}$ and $\hat{\kappa}_{1}$ in upper left part of Figure 5. The separatrix $C$ lifts to a plane in the $3 \mathrm{D}$ system

$$
\hat{C}:=\{(\hat{x}, y, \hat{z}) \mid y=C(x), \hat{z} \text { arbitrary. }\}
$$


where the function $C$ is defined in (16). This plane intersects wall $W_{1}=$ $\left[0, \theta_{z x}\right] \times \theta_{x z} \times\left[\theta_{x y}, 2\right]$, see Figures 5-6, between the cells $\hat{\kappa}_{0}$ and $\hat{\kappa}_{1}$. We will choose $W_{1}$ as a Poincaré section and study the return map to this wall.

We first observe that $W_{1}$ is a transparent wall and trajectories starting at $W_{1}$ will exit $\hat{\kappa}_{1}$ either through the wall $W_{2}=\theta_{z x} \times\left[0, \theta_{x z}\right] \times\left[\theta_{x y}, 2\right]$ or through the wall $W_{3}=\left[0, \theta_{z x}\right] \times\left[0, \theta_{x z}\right] \times \theta_{x y}$.

We solve (17) with initial conditions $\left(\hat{x}(0), \theta_{x z}, \hat{y}(0)\right) \in W_{1}$ to get

$$
\begin{aligned}
& \hat{x}(t)=\gamma_{x}^{-1}+\left(\hat{x}(0)-\gamma_{x}^{-1}\right) e^{-\gamma_{x} t} \\
& \hat{z}(t)=\theta_{x z} e^{-\gamma_{z} t} \\
& y(t)=\gamma_{y}^{-1}+\left(y(0)-\gamma_{y}^{-1}\right) e^{-\gamma_{y} t} .
\end{aligned}
$$

Let $T_{x}>0$ and $T_{y}>0$ be times when solution (20) reaches the thresholds corresponding to $W_{2}$ and $W_{3}$, respectively: $\hat{x}\left(T_{x}\right)=\theta_{z x}$ and $\hat{y}\left(T_{y}\right)=\theta_{x y}$. Then, the condition $T_{x}=T_{y}$ defines a line of initial conditions (separatrix) in $W_{1}$ that separates solutions that will enter $W_{2}$ (above the separatrix) and $W_{3}$ (below the separatrix):

$$
e^{-T_{x}}=\left(\frac{\theta_{z x}-\gamma_{x}^{-1}}{\hat{x}(0)-\gamma_{x}^{-1}}\right)^{\gamma_{x}^{-1}}=\left(\frac{\theta_{x y}-\gamma_{y}^{-1}}{\hat{y}(0)-\gamma_{y}^{-1}}\right)^{\gamma_{y}^{-1}}=e^{-T_{y}}
$$

Note that this defines the identical equation to (16), recalling that $\theta_{z x}=$ $\theta_{x x}$. Therefore we have the following lemma.

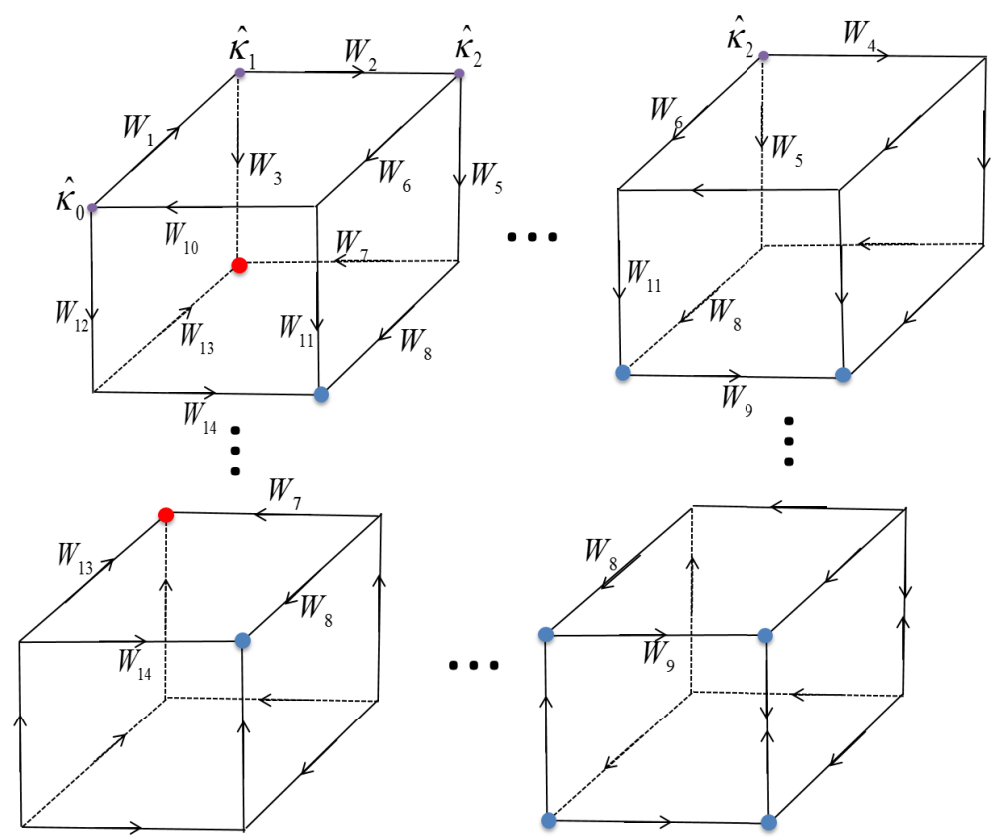

Fig. 6: An "exploded" state transition diagram to clarify the labeling of walls $W_{i}, i=$ $1, \ldots, 14$ in Figure 5 . 
Lemma 55 The separatrix $S:=\hat{C} \cap W_{1}$ has identical equations to the $2 D$ separatrix $C$, and therefore does not depend on value of $\epsilon$. An initial condition starting on $W_{1}$ above $S$ enters the wall $W_{2}$. An initial condition on $W_{1}$ starting below $S$ enters the wall $W_{3}$ and converges to $\hat{A}$.

Proof The only thing that remains to be shown is that a solution crossing the wall $W_{3}$ converges to $\hat{A}$. This follows from Figure 5 , the fact that $W_{3}$ is a transparent wall, and that $\hat{A}$ attracts all trajectories within its cell.

In Figure 7 (a) we show the separatrix $S$ on $W_{1}$. We color the region in the basin of attraction of $\hat{A}$ by red; the solutions entering $W_{2}$ are colored gray.

In what follows, we will describe the Poincaré map on $W_{1}$ by tracing possible trajectories through the state transition diagram in Figure 5. Passing through each cell, each entrance wall is partitioned by separatrices that divide initial conditions into sets that pass through the same exit wall of the adjacent cell. These can be computed by equating the times that it takes for an initial condition to reach various thresholds containing the exit walls; separatrices correspond to solutions that exit the cell through a one-dimensional intersection of two exit walls. These calculations are completely analogous to the calculations leading to separatrix $S$ above and will be omitted.

We start with wall $W_{2}$ that is an entrance wall of cell $\hat{\kappa}_{2}=\left[\theta_{z x}, \theta_{y x}\right] \times$ $\left[0, \theta_{x z}\right] \times\left[\theta_{x y}, 2\right]$. There are three exit walls $W_{4}, W_{5}$ and $W_{6}$. There are three separatrices: between solutions entering $W_{5}$ and $W_{6}$, between those entering $W_{4}$ and $W_{5}$ and those that enter $W_{4}$ and $W_{6}$.

Because of the limitation of the domain to $\hat{x}, y \leq 2$, it turns out that there are no initial conditions on $W_{2}$ that reach $W_{4}$. Therefore, we only consider the separatrix between $W_{5}$ and $W_{6}$. In Figure 7 (b), we show the preimage of this separatrix on wall $W_{1}$, above the original separatrix. The red region on the bottom is the same path $W_{1}$ to $W_{3}$ as in Figure 7 (a). The new separatrix divides the path $W_{1}, W_{2}, W_{6}$ (the large topmost gray region) from $W_{1}, W_{2}, W_{5}$ (the middle gray region). Both regions are gray, because neither path has determined global dynamics at this point.

Consider now the wall $W_{5}$. There are two exit walls $W_{7}$ and $W_{8}$ and therefore there is a separatrix on $W_{5}$ that separates initial data that leads to these regions. Importantly, the solutions that enter $W_{7}$ will converge to $\hat{A}$ since $W_{7}$ is transparent and $\hat{A}$ attracts all solutions within its cell. At the same time, solutions that enter $W_{8}$ must converge to $\hat{B}$ since $W_{8}$ is transparent and borders the trapping region $\hat{B}$. The preimage of this separatrix on $W_{1}$ splits the middle gray strip in Figure 7 (b) into two parts; the bottom part will execute the trajectory $W_{2}, W_{5}, W_{7}$ and converge to $\hat{A}$, the top part will execute $W_{2}, W_{5}, W_{8}$ and converge to $\hat{B}$, as shown in Figure 7 (c).

Similar discussion on $W_{6}$ will produce the preimage on $W_{1}$ of a separatrix which splits solutions that lead to wall $W_{11}$ and thus to region $\hat{B}$ (next blue stripe in Figure $7(\mathrm{~d}))$ and those that come to wall $W_{10}$. There the next split separates those solutions that go back to $W_{1}$ (large gray region in Figure 7 (d)) and those that go down to $W_{12}$. The final split separates solutions going through wall $W_{13}$ to $\hat{A}$ and those that go through wall $W_{14}$ to $\hat{B}$. 


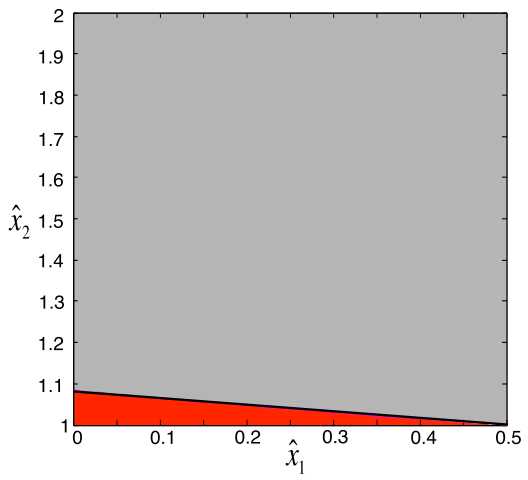

(a)

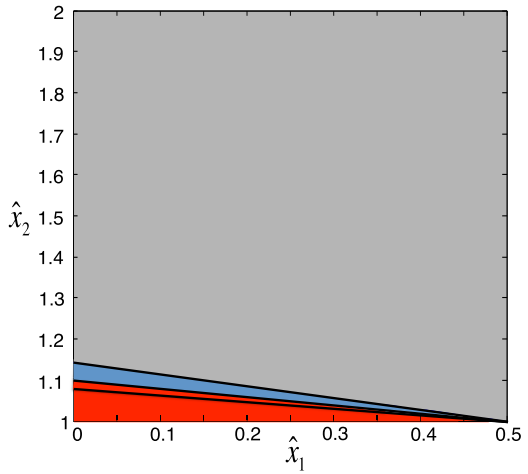

(c)

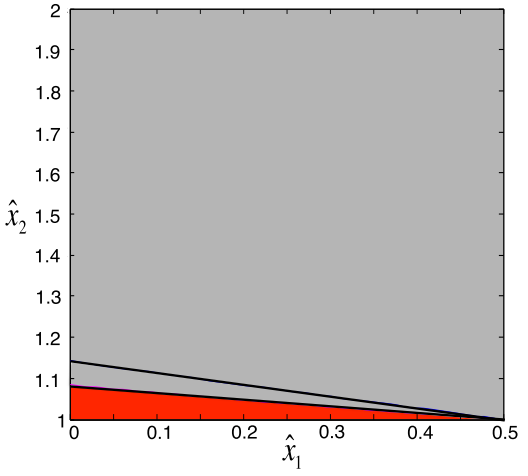

(b)

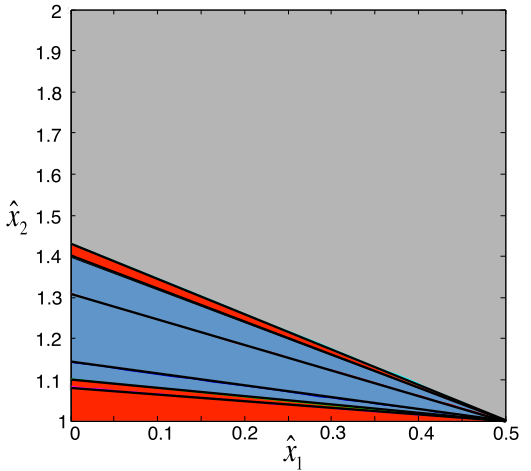

(d)

Fig. 7: Red shading $=$ path to steady state $\hat{A}$; blue shading $=$ path to trapping region $\hat{B}$; gray shading $=$ undetermined global dynamics.

(a) The separatrix on the Poincaré section $W_{1}$. Everything below goes to $W_{3}$ and everything above goes to $W_{2}$

(b) Two separatrices in the Poincare section $W_{1}$ after two steps. From top to bottom, the paths are $W_{1} \rightarrow W_{2} \rightarrow W_{6}, W_{1} \rightarrow W_{2} \rightarrow W_{5}$, and $W_{1} \rightarrow W_{3}$.

(c) Refining the map forward from $W_{5}$. From top to bottom: $W_{1} \rightarrow W_{2} \rightarrow W_{6}$

$W_{1} \rightarrow W_{2} \rightarrow W_{5} \rightarrow W_{8}, W_{1} \rightarrow W_{2} \rightarrow W_{5} \rightarrow W_{7}$, and $W_{1} \rightarrow W_{3}$.

(d) Refining the map forward from $W_{6}$. From top to bottom:

Gray: $\quad W_{1} \rightarrow W_{2} \rightarrow W_{6} \rightarrow W_{10} \rightarrow W_{1}$

Red: $\quad W_{1} \rightarrow W_{2} \rightarrow W_{6} \rightarrow W_{10} \rightarrow W_{12} \rightarrow W_{13}$

Blue: $\quad W_{1} \rightarrow W_{2} \rightarrow W_{6} \rightarrow W_{10} \rightarrow W_{12} \rightarrow W_{14}$

Blue: $\quad W_{1} \rightarrow W_{2} \rightarrow W_{6} \rightarrow W_{11}$

Blue: $\quad W_{1} \rightarrow W_{2} \rightarrow W_{5} \rightarrow W_{8}$

Red: $\quad W_{1} \rightarrow W_{2} \rightarrow W_{5} \rightarrow W_{7}$

Red: $\quad W_{1} \rightarrow W_{3}$ 
Let $D \subset W_{1}$ be the region of $W_{1}$ separated into red and blue colored stripes in Figure 7 (d). The initial data in this region will not return to $W_{1}$ and so they are not part of the domain of the Poincaré map. In particular, the Poincaré map is a flow-defined homeomorphism

$$
\tilde{P}: W_{1} \backslash D \rightarrow W_{1}
$$

Notice that since $y \in\left(\theta_{x, y}, 2\right]$ and $\hat{z}=\theta_{x, z}$ on $W_{1}$, the wall $W_{1}$ corresponds to the Poincaré section $\hat{S}_{2}$ given in (31). Therefore, the results of Section 6.3 hold; in particular, $W_{1}$ admits the same decomposition as $\hat{S}_{2}$ and is a contraction map. In fact, the $\hat{x}$ component of the Poincaré map $\tilde{P}_{x}=g_{2} \circ g_{1}$ is known explicitly, where the $g_{i}$ are defined in (34) and (37).

Lemma 56 Consider the flow generated by (17) for any $\epsilon>0$. Then there are an infinite number of disjoint open sets $\left\{A_{n}\right\}_{n=1}^{\infty},\left\{B_{n}\right\}_{n=1}^{\infty} A_{n}, B_{n} \subset W_{1} \backslash D$, such that

- if initial data $\left(\hat{x}(0), y(0), \theta_{z}\right) \in A_{n}$ then the solution converges to steady state $\hat{A}$;

- if initial data $\left(\hat{x}(0), y(0), \theta_{z}\right) \in B_{n}$ then the solution converges to trapping region $\hat{B}$.

Proof Let $A_{0} \subset W_{1}$ be the domain that corresponds to the path $\left(W_{1}, W_{2}, W_{6}, W_{10}, W_{12}, W_{13}\right)$, which is the top red cone in Figure $7(\mathrm{~d})$. Let $B_{0} \subset W_{1}$ be the blue stripe below $A_{0}$ corresponding to the path $\left(W_{1}, W_{2}, W_{5}, W_{8}\right)$ (either of the other two blue stripes would work as well). Note that both of these domains are open, being preimages of open sets by a finite number of flow-defined maps. Both lie above the separatrix $S$.

Define

$$
A_{n}:=\tilde{P}^{-n}\left(A_{0}\right), \quad \text { and } \quad B_{n}:=\tilde{P}^{-n}\left(B_{0}\right) .
$$

Clearly these sets are again open and disjoint, since $\tilde{P}$ is a homeomorphism.

We now describe in more detail the geometry of the problem. The Poincare map $\tilde{P}$ is defined using the flow in the four cells that share the walls $W_{1}, W_{2}, W_{6}$ and $W_{10}$ where $\dot{y}<0$. At the same time, the variables $x$ and $z$ oscillate around the axis that is an intersection of these four cells. Figure 7 (d) reflects that: the solutions that start with a higher initial value of $y(0)$ stay in the set of cells longer and so stripes that are higher have a longer trajectory among $W_{1}, W_{2}, W_{6}, W_{10}$. Furthermore, the gray region maps onto all of $W_{1}$, and, in particular, the set $D$. Therefore the strip $A_{n}$ consists of initial data giving rise to solutions that return to $W_{1}$ exactly $n$ times (each time executing a path $\left(W_{1}, W_{2}, W_{6}, W_{10}\right)$, before landing in $A_{0}$ and subsequently executing a path $\left(W_{1}, W_{2}, W_{6}, W_{10}, W_{12}, W_{13}\right)$. Similarly, the strip $B_{n}$ consists of initial data giving rise to solutions that return to $W_{1}$ exactly $n$ times (each time executing a path $\left.\left(W_{1}, W_{2}, W_{6}, W_{10}\right)\right)$, before landing in $B_{0}$ and subsequently executing a path $\left(W_{1}, W_{2}, W_{5}, W_{8}\right)$. 
Theorem 57 Let $\hat{I}^{0} \in W_{1}$ be an initial condition above the separatrix $S$. Then for any $\epsilon_{0}$ there is an infinite sequence of intervals

$$
I_{1}^{A}>I_{1}^{B}>I_{2}^{A}>I_{2}^{B}>\ldots \subset\left(0, \epsilon_{0}\right], \text { or } I_{1}^{B}>I_{1}^{A}>I_{2}^{B}>I_{2}^{A}>\ldots \subset\left(0, \epsilon_{0}\right] \text {, }
$$

where $I>J$ means $x>y$ for all $x \in I, y \in J$, such that if $\epsilon \in I_{j}^{A}$ then the solution of (17) starting at $\hat{I}^{0}$ converges to $A$, while when $\epsilon \in I_{j}^{B}$ then the solution of (17) starting at $\hat{I}^{0}$ converges to $B$.

Proof Fix $\hat{I}^{0} \in W_{1}$ above $S$. Then the trajectory starting at $\hat{I}^{0}$ undergoes $n$ oscillations in $x, z$ variables before crossing threshold $y=\theta_{x, y}$ for some $n \geq 0$. The time $T_{y}$ to the exit wall $y=\theta_{x, y}$ is fixed by the initial condition $\hat{I}^{0}$ and does not depend on $\epsilon$. However, $\dot{\hat{z}}$ increases with $\epsilon$ and this the trajectory starting at $\hat{I}^{0}$ executes more $x, z$ oscillations in the fixed time $T_{y}$. To see this, recall the $n$-th return time of the Poincaré map $P$ in (41). $\tilde{P}$ has the return time of the form (41), which we rewrite here for convenience:

$$
\sum_{i=1}^{n} T_{R}^{i}=T_{x}^{1}+\sum_{i=1}^{n} f(\epsilon ; i) \quad \text { with } \lim _{\epsilon \rightarrow 0} f(\epsilon ; i)=0 .
$$

Since $T_{y}-T_{x}^{1}$ is fixed by initial data, it follows that for every $\epsilon>0$ there is $N=N(\epsilon)$ such that

$$
\sum_{i=1}^{N(\epsilon)} f(\epsilon ; i) \leq T_{y}-T_{x}^{1}<\sum_{i=1}^{N(\epsilon)+1} f(\epsilon, i+1) .
$$

It follows that

$$
\lim _{\epsilon \rightarrow 0} N(\epsilon)=\infty
$$

and thus the number of wall crossings increases. This means that the preimages of the separatrices in $W_{1}$ will sweep across $\hat{I}^{0}$ in a continuous manner as $\epsilon \rightarrow 0$, so that alternating $A_{i}$ and $B_{i}$ stripes will contain $\hat{I}^{0}$. Also by continuity and the fact that the stripes $A_{n}$ and $B_{n}$ are open, there will be a range of $\epsilon$ when $\hat{I}^{0} \in A_{n}(\epsilon)$ as well as range of $\epsilon$ when $\hat{I}^{0} \in B_{n}(\epsilon)$, for all $n$ sufficiently large.

We conclude that the global dynamics of the systems (13) and (17) are different. In the domain $\kappa_{1}$ for (13) a single separatrix $C$ separates basins of attraction of $A$ and $B$. Above $C$, all solutions converge to $B$ as determined by perturbation analysis with $q>0$. In contrast, for any $\epsilon>0$ in (17), there are infinitely many domains above the corresponding separatrix $S=\hat{C} \cap W_{1}$ where solutions converge to $\hat{A}$. When we examine the limit of solutions of (17) as $\epsilon \rightarrow 0$ for a fixed initial condition, Theorem 57 shows that such a limit does not exist. This shows that the prediction of the dynamics of (13) using a perturbation $q>0$ gives different results than a perturbation $\epsilon>0$. 


\section{Discussion}

We have discussed both local and global changes in dynamics that occur due to perturbing switching systems with black and white walls (proteinonly systems) into higher dimensional systems with fast-slow dynamics (posttranslationally modified protein or PTM systems). The extended system has no black and white walls, but the new variables introduce a short negative feedback loop for each negatively self-regulated protein. We explore the consequences of this negative loop on the ability of solutions of a PTM system to shadow trajectories of a protein-only system near a black wall, as well as differences in the asymptotic dynamics of the two systems. The dynamics near the perturbed white wall should be described by positive feedback between the original protein and the modified protein, which we do not explore in this paper.

We first studied changes to the dynamics on a local scale, by prescribing a small $\delta$-neighborhood as a target for the trajectory of the PTM system after a given finite time. The perturbation to $\epsilon>0$ is not smooth, and therefore the theorem of Tikhonov [26] is not available to us. However, we proved that for a given initial condition and prescribed $\delta>0$, there is an $\epsilon_{0}>0$ such that for all $\epsilon<\epsilon_{0}$, the trajectory beginning at the given initial condition shadows the protein-only solution in a neighborhood about a black wall. Furthermore, we showed that for any fixed $\delta>0$ and $\epsilon>0$, there is a region of initial conditions that is excluded in the sense that a trajectory arising from an initial condition within the excluded region will lie outside of the $\delta$-neighborhood at the desired time. The excluded region has a nontrivial, complex structure induced by a rotating vector field, which is itself induced by the fast dynamics introduced by the perturbation. These excluded regions may impact the global dynamics of a system, although the example we discuss in Section 5 does not examine the local excluded regions and instead focuses on basins of attraction of global attractors.

After proving the general local result for the existence of an excluded region, we then moved on to an examination of global dynamics using an example system from [18]. We defined the dynamics of the two-dimensional switching system (13) to be the limit of smooth Hill functions as $q \rightarrow 0$, and discovered the existence of a separatrix in cell $\kappa_{1}$ (see Figure 4). We then compared these dynamics with a perturbation into a three-dimensional system (17) with a small parameter $\epsilon>0$. We found the corresponding location of the separatrix in the 3D system and showed that there are an infinite number of separatrices lying above the original separatrix. Thus the dynamics are quite different between the two systems for any finite $\epsilon$.

While we did not analyze in full generality the case when both $q>0$ and $\epsilon>0$, we will briefly discuss this situation in the context of the system (17) with a fixed $\epsilon>0$. Pick a point $\hat{I}^{0}$ in the interior of a red or blue triangular region in $W_{1}$ in Figure 7 (d). Then the solution under $q=0$ flow that starts at $\hat{I}^{0}$ has to cross a finite number of transparent walls before it arrives in an open basin of attraction of $\hat{A}$ or $\hat{B}$. From Remark 36 , we know that this solution can 
be perturbed into a $q>0$ flow that tends to the same basin of attraction. By continuity, there is an open neighborhood about $\hat{I}^{0}$ with the same property. This shows that for any open set $U$ of initial data in the interior of any cone (as for example in Figure $7(\mathrm{~d}))$ there is a $q_{1}=q_{1}(U, \epsilon)$, such that for $q<q_{1}$ the $q$-solution will shadow the solution of the switching system (17). When the cone is associated to a longer path before entering a basin of $\hat{A}$ or $\hat{B}$, the choice of $q_{1}$ will be smaller, but at least a finite number of alternating open regions of attraction will exist for any $q_{1}<<1$ fixed. Thus for each $\epsilon>0$ there is $q_{1}=q_{1}(\epsilon)$ such that are at least a finite number of domains of attraction of $\hat{A}$ within what would be a domain of attraction of $B$ in the two-dimensional system (13) for $0<q<q_{1}$.

We summarize our results with the help of Figure 8, which depicts two types of perturbations of the switching system. The $2 \mathrm{D}$ switching system analyzed above lies on the intersection $q=0, \epsilon=0$. We used a perturbation $q>0$ with $\epsilon=0$ fixed (vertical axis) followed by taking $\lim _{q \rightarrow 0}$ to decide that the solutions with initial data above the separatrix $C$ converge to the attractor $B$. However, when we analyzed the $\epsilon$-perturbation with $q=0$ (horizontal axis) we discovered that we cannot assign convergence to either $\hat{A}$ or $\hat{B}$ with the same initial data, since for any initial data the convergence assignment continues to flip between $\hat{A}$ and $\hat{B}$ as $\epsilon \rightarrow 0$. Thus these two perturbations resolve the black wall dynamics in different ways. Finally, we show that the interwoven basins of attraction observed at $\epsilon>0, q=0$ perturb to the $\epsilon>0, q>0$ case, for sufficiently small $q$. Here the size of admissible $q$ depends on $\epsilon$, and we speculate that it most likely converges to zero $\lim _{\epsilon \rightarrow 0} q_{1}(\epsilon)=0$, as we depict in the light gray rectangle in Figure 8.

The observations in the previous paragraph are seemingly at odds with the results of Edwards et al. [5]; however on closer inspection we discover that the two results are compatible. In [5], the limit $\epsilon \rightarrow 0$ for $q=q_{0}>0$ fixed is analyzed using singular perturbation theory for a general PTM system. They found that the limit exists for a finite time $T$, albeit with a steeper sigmoid that is not a Hill function. This means that when $(q>0, \epsilon=0)$, there is a perturbation into $\epsilon>0$ with a well-defined limit. So in our example in Section 5 , the result of [5] would predict that there is a separatrix between $\hat{A}$ and $\hat{B}$ on $W_{1}$ when $(q>0, \epsilon>0)$. However, we have just argued that the limit of the basins of attraction as $\epsilon \rightarrow 0$ with $q=q_{0}$ fixed are not well-defined, and in fact that when $(q=0, \epsilon>0)$, there is a perturbation into $q>0$ in which the basins of attraction of $\hat{A}$ and $\hat{B}$ alternate multiple times on $W_{1}$. These two disparate results can be reconciled by suggesting that there is a region in $(q, \epsilon)$ space where the behavior of the limit transitions between a separatrix and alternating basins, as depicted in Figure 8. The dark gray triangle represents the perturbation into $\epsilon>0$ from a $(q>0, \epsilon=0)$ system, and the light gray triangle represents the $q>0$ perturbation for a $(q=0, \epsilon>0)$ system. The critical message is that when there are two small time scales governing a fastslow system, the relative magnitude of the time scales determines the behavior of the system. 


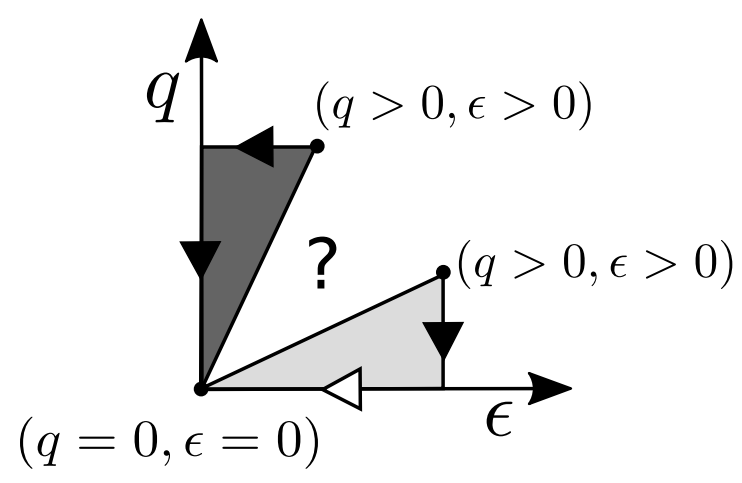

Fig. 8: Schematic of the two perturbations in $(q, \epsilon)$ space, with $(\epsilon \geq 0, q=0)$ on the horizontal axis and $(\epsilon=0, q \geq 0)$ on the vertical axis. The dark gray triangle depicts the perturbation into $\epsilon>0$, and the light gray triangle depicts the perturbation into $q>0$. Black arrows represent well-defined limits, and the white arrow represents an ill-defined limit. The blank area in the center has unknown limiting behavior.

Alternating basins of attraction for global attractors have been seen in other switching systems that do not consider $\epsilon$ perturbations as in this paper. Edwards and collaborators $[4,14]$ investigated systems in which thresholds can regulate multiple proteins at once, a relaxation of our Assumption 21. The fast systems that arise in these situations for small $q>0$ occur near walls, when one or more variables exists on the steep portion of a sigmoid function. In [14], a $4 \mathrm{D}$ system is constructed that has a limit cycle when three of the variables are switching, that is, near thresholds. When $q=0$, the limit cycle spirals into a 1D "black line" in finite time and slides along it until the intersection of all four thresholds. For small $q>0$, the three variables maintain a limit cycle as they approach the intersection of all four thresholds. Depending on the initial condition, the trajectory will land in one of two basins of attraction for different attractors. The authors describe this phenomenon as sensitive dependence on initial conditions, and note that the sensitivity increases with decreasing $q$ (see especially Figure 5 in [14] and compare it our Theorem 57).

Even though [14] explores a different system than the one we study, the key point is that oscillations induced by a perturbation can affect the global dynamics of a system. However, there is a further similarity between our work and that of [14]. In the 3D system (17), there is a "black line" like the one in [14], described by $\left\{\theta_{z x}\right\} \times\left(\theta_{x y}, 2\right] \times\left\{\theta_{x z}\right\}$. The $(\hat{x}(t), \hat{z}(t))$ solution spirals downward and inward about this line until it crosses the plane $y=\theta_{x y}$. At this point it enters either the $\hat{A}$ or $\hat{B}$ basin of attraction. (We remark that unlike [14], $(\hat{x}(t), \hat{z}(t))$ only approaches $\left(\theta_{z x}, \theta_{x z}\right)$ in infinite time, not finite time, since we do not allow multiple proteins to be regulated at one threshold.) The similar geometry of these two disparate problems may be indicative of a more general process than has been described in this paper. 
Our work opens up many interesting questions. The most important one is to understand if our example presents an aberration, or a rule for the switching systems. In other words, are there some easily identifiable signatures of the switching network that would allow us decide if the removal of the black walls by adding additional switching variables will change the global dynamics? In the first part we have shown that the shadowing property of the switching system by the perturbed system is not uniform in the initial condition. This is a general property of this type of perturbation. But under what circumstances this leads to changes in global dynamics is not clear.

In the analysis of our example we observed a complex behavior emerging after an $\epsilon>0$ perturbation of a system where a black wall attached to a white wall. Perhaps if we only allow a black wall to be attached to a transparent wall, the $\epsilon>0$ perturbation will be more tame. Another set of questions relate to complete elucidation of interaction between $q$ and $\epsilon$ perturbations, as shown in Figure 8. What happens to the domain of convergence to $\hat{B}$ that exists for $\epsilon>0, q>0$, as we fix $q$ and let $\epsilon \rightarrow 0$ ? Are there results for general systems along this venue, rather than for a particular example? We will leave these questions for future investigations.

Lastly, what is the implication of our work for modeling biological systems? We show that removing a black wall in the switching system by adding an additional variable should be done with caution; the resulting dynamics may not agree with those of the original system, where the dynamics on the black wall are defined as a limit of small perturbations. This disagreement extends to prediction of global dynamics. Therefore, when a modeler choses between these models the deciding factor should be not an ease of analysis, but the type of dynamics that these models produce. They may not be the same. From a broader perspective, our results should encourage us to seek better modeling approaches where the model predictions are coarser, but also more robust to perturbations in parameters and the types of models used. Since the coarseness of available biological data may not be able to distinguish between models whose predictions differ on a fine scale, model predictions should perhaps be adjusted to match the coarseness of the data.

\section{References}

1. Bornholt, S.: Boolean network models of cellular regulation: prospects and limitations. J. R. Soc. Interface 2008 5, 134-150 (2008)

2. Casey, R., Jong, H.D., Gouzé, J.: Piecewise-linear Models of Genetic Regulatory Networks: Equilibria and their Stability. Journal of mathematical biology 56, 27-56 (2006). DOI 10.1007/s00285-005-0338-2. URL http://link.springer.com/article/10.1007/s00285-005-0338-2

3. Edwards, R.: Analysis of continuous-time switching networks. Physica D: Nonlinear Phenomena 146(March), 165-199 (2000). URL http://www.sciencedirect.com/science/article/pii/S0167278900001305 
4. Edwards, R., Hill, A., Jacquier, M.: Analysis of transient damped oscillations in gene regulatory networks. In: 21st International Symposium on Mathematical Theory of Networks and Systems, pp. 487-489. Groningen, The Netherlands (2014)

5. Edwards, R., Machina, a., McGregor, G., van den Driessche, P.: A Modelling Framework for Gene Regulatory Networks Including Transcription and Translation. Bulletin of Mathematical Biology (2015). DOI 10.1007/s11538-015-0073-9. URL http://link.springer.com/10.1007/s11538-015-0073-9

6. Gedeon, T., Harker, S., Kokubu, H., Mischaikow, K., Oka, H.: Global dynamics for steep sigmoidal nonlinearities in two dimensions. Submitted to Physica D (2015), ArXiv 1508.02438

7. Glass, L., Pasternack, J.: Stable oscillations in mathematical models of biological control systems. Journal of Mathematical Biology 6, 207-223 (1978). URL http://link.springer.com/article/10.1007/BF02547797

8. Goncalves, E., Bucher, J., Ryll, A., Niklas, J., Mauch, K., Klamt, S., Rocha, M., Saez-Rodriguez, J.: Bridging the layers: towards integration of signal transduction, regulation and metabolism into mathematical models. Mol. BioSyst. 9, 1576-1583 (2013). DOI 10.1039/C3MB25489E. URL http://dx.doi.org/10.1039/C3MB25489E

9. Gouzé, J.L., Sari, T.: A class of piecewise linear differential equations arising in biological models. Dynamical Systems 17(4), 299-316 (2002). DOI 10.1080/1468936021000041681. URL http://www.tandfonline.com/doi/abs/10.1080/1468936021000041681

10. Heatha, A., Kavria, L.: Computational challenges in systems biology. Computer Science Review 3, 1-17 (2009)

11. Ironi, L., Panzeri, L., Plahte, E., Simoncini, V.: Dynamics of actively regulated gene networks. Physica D: Nonlinear Phenomena 240(8), 779-794 (2011). DOI 10.1016/j.physd.2010.12.010. URL http://linkinghub.elsevier.com/retrieve/pii/S016727891000360X

12. Karlebach, G., Shamir, R.: Modelling and analysis of gene regulatory networks. Nature 9(770) (2008)

13. Machado, D., Costa, R., Rocha, M., Ferreira, E., Tidor, B., Rocha, I.: Modeling formalisms in systems biology. AMB Express 1(1), 45 (2011). DOI 10.1186/2191-0855-1-45. URL http://www.ambexpress.com/content/1/1/45

14. Machina, A., Edwards, R., van den Driessche, P.: Sensitive dependence on initial conditions in gene networks. Chaos 23, 025,101-1-025,101-9 (2013). DOI 10.1063/1.4807480

15. Machina, A., Ponosov, A.: Filippov solutions in the analysis of piecewise linear models describing gene regulatory networks. Nonlinear Analysis: Theory, Methods \& Applications 74(3), 882-900 (2011). DOI 10.1016/j.na.2010.09.039. URL http://linkinghub.elsevier.com/retrieve/pii/S0362546X10006620

16. Mestl, T., Plahte, E., Omholt, S.W.: A mathematical framework for describing and analysing gene regulatory networks. Journal of theoreti- 
cal biology 176(2), 291-300 (1995). DOI 10.1006/jtbi.1995.0199. URL http://www.ncbi.nlm.nih.gov/pubmed/7475117

17. Ott, E.: Chaos in dynamical systems, second edn. Cambridge University Press, Cambridge (2002). DOI 10.1017/CBO9780511803260. URL http://dx.doi.org.proxy.libraries.rutgers.edu/10.1017/CBO9780511803260

18. Plahte, E., Kjøglum, S.: Analysis and generic properties of gene regulatory networks with graded response functions. Physica D: Nonlinear Phenomena 201(1-2), 150-176 (2005). DOI 10.1016/j.physd.2004.11.014. URL http://linkinghub.elsevier.com/retrieve/pii/S0167278904004531

19. Plahte, E., Mestl, T., Omholt, S.W.: A methodological basis for description and analysis of systems with complex switch-like interactions. Journal of mathematical biology 36(4), 321-48 (1998). URL http://www.ncbi.nlm.nih.gov/pubmed/9579029

20. Saadatpour, A., Reka, A.: Boolean modeling of biological regulatory networks: A methodology tutorial. Methods 62(5), 3-12 (2013)

21. Storch, K.F., Lipan, O., Leykin, I., Viswanathan, N., Davis, F.C., Wong, W.H., Weitz, C.J.: Extensive and divergent circadian gene expression in liver and heart. Nature 417(6884), 78 (2002)

22. Thomas, R.: Boolean formalization of genetic control circuits. Journal of Theoretical Biology 42, 563-585 (1973). DOI 10.1016/00225193(73)90247-6

23. Thomas, R.: Regulatory networks seen as asynchronous automata: A logical description. Journal of Theoretical Biology 153, 1-23 (1991). DOI 10.1016/S0022-5193(05)80350-9

24. Tyson, J.J., Novak, B.: Chapter 14 - irreversible transitions, bistability and checkpoint controls in the eukaryotic cell cycle: A systemslevel understanding. In: A.M.W.V. Dekker (ed.) Handbook of Systems Biology, pp. 265 - 285. Academic Press, San Diego (2013). DOI http://dx.doi.org/10.1016/B978-0-12-385944-0.00014-9. URL http://www.sciencedirect.com/science/article/pii/B9780123859440000149

25. Veflingstad, S.R., Plahte, E.: Analysis of gene regulatory network models with graded and binary transcriptional responses. Biosystems 90, 323-339 (2007). DOI 10.1016/j.physd.2004.11.014. URL http://linkinghub.elsevier.com/retrieve/pii/S0167278904004531

26. Wasow, W.: Asymptotic expansions for ordinary differential equations. Interscience Publishers (1965)

\section{Appendix}

We begin by proving Lemma 42 , which is an immediate consequence of Lemmas 61 and 62 below.

Lemma 61 Consider a black wall $w(x, x)$ with an exit hyperplane $y_{J}=\theta_{x, J}$ and flanking cells $\kappa$ and $\bar{\kappa}$ such that $\kappa \cap \bar{\kappa}=w(x, x)$. Define $e(x, J):=\left\{y_{J}=\right.$ $\left.\theta_{x, J}\right\} \cap \operatorname{int}(\kappa \cup \bar{\kappa})$. Then there exists a nonempty, compact, rectangular region 
$\mathcal{R} \subset \kappa \cup \bar{\kappa}$ such that $\mathcal{R} \cap w(x, x) \cap e(x, J) \neq \emptyset$ and for every $I^{0} \in \mathcal{R}$, there exists $T_{I^{0}} \geq 0$ such that $\left(x(t), y_{i}(t)\right) \in \mathcal{R}$ for $t \in\left[0, T_{I^{0}}\right)$ and $\left(x\left(T_{I^{0}}\right), y_{i}\left(T_{I^{0}}\right)\right) \in$ $\mathcal{R} \cap e(x, J)$.

Proof We first observe that both the black wall $w(x, x)$ and the exit hyperplane $y_{J}=\theta_{x, J}$ must lie on the boundary of $\kappa$ and on the boundary of $\bar{\kappa}$. Therefore by Definition 39, the definition of an exit hyperplane, and Definition 34, describing the flow near a black wall, there exists a neighborhood $V$ of $e(x, J)$ such that

- $V \cap$ int $\kappa, V \cap$ int $\bar{\kappa}$, and $V \cap w(x, x)$ are all relatively open and nonempty;

- for any $I^{0} \in V \cap \kappa$ or $I^{0} \in V \cap \bar{\kappa}$, the solution starting at $I^{0}$ exits $\kappa$ or $\bar{\kappa}$ either through $\left\{y_{J}=\theta_{x, J}\right\}$ or through $w(x, x)$;

- for any $I^{0} \in V \cap w(x, x)$ the solution starting at $I^{0}$ exits $w(x, x)$ through $w(x, x) \cap e(x, J)$.

Consider three flows: $\varphi_{\kappa}$ on $\kappa$ and $\varphi_{\bar{\kappa}}$ on $\bar{\kappa}$ from (8) and $\varphi_{w(x, x)}$ on $w(x, x)$ defined in (7). Since the latter flow is defined as restriction of both $\varphi_{\kappa}$ and $\varphi_{\bar{\kappa}}$, their union defines a continuous flow $\tilde{\varphi}$ on $V \cap(\kappa \cup \bar{\kappa} \cup w(x, x))$.

Take $I^{0} \in w(x, x) \cap e(x, J)$ such that the flow $\varphi_{w(x, x)}$ is transversal to $e(x, J)$. Such a point must exist by the assumption that $\left\{y_{J}=\theta_{x, J}\right\}$ is an exit wall for $w(x, x)$. Since the flow on the black wall (7) is a restriction of $\varphi_{\kappa}$ and $\varphi_{\bar{\kappa}}$, there is a neighborhood of $I^{0}$

$$
\tilde{\mathcal{R}}:=\mathcal{R}_{x} \times \mathcal{R}_{y_{1}} \times \ldots \mathcal{R}_{y_{J-1}} \times \theta_{x, J} \times \mathcal{R}_{y_{J+1}} \times \ldots \times \mathcal{R}_{y_{n-1}} \subset e(x, J) \cap V,
$$

where $\mathcal{R}_{v}$ is an interval in the variable $v$, such that the flow $\tilde{\varphi}$ is transversal to $e(x, J)$.

By continuity of $\tilde{\varphi}$ on $V$ and transversality of $\tilde{\varphi}$ on $\tilde{\mathcal{R}}$, there is an interval $\mathcal{R}_{y_{J}}$ in variable $y_{J}$ such that all solutions in

$$
\mathcal{R}:=\mathcal{R}_{y_{J}} \times \tilde{\mathcal{R}}=\mathcal{R}_{x} \times \mathcal{R}_{y_{J}} \times \prod_{i \neq J} \mathcal{R}_{y_{i}}
$$

exit $\mathcal{R}$ directly through $\tilde{\mathcal{R}} \backslash w(x, x)$, or enter the black wall $w(x, x)$ and exit $\mathcal{R}$ through $w(x, x) \cap e(x, J)$.

When we add the modified protein $\hat{z}$ to the protein-only system, the $n-1$ dimensional black wall $w(x, x)$ where $x=\theta_{x}$ disappears. The analogous region to $w(x, x)$ in the PTM system (9) is the set of two transparent walls

$$
\mathcal{W}:=\left\{\left(\hat{x}, \hat{y}_{i}, \hat{z}\right) \mid\left(\hat{x}, \hat{y}_{i}\right) \in w(x, x), \hat{z} \in\left[\theta_{z}-\beta_{z}, \theta_{z}+\beta_{z}\right]\right\}
$$

Notice that this set is one dimension higher than $w(x, x)$. We now show that there exists a region $\hat{\mathcal{R}}(\epsilon)$ intersecting $\mathcal{W}$, analogous to $\mathcal{R}$, where only $\hat{x}, \hat{z}$, and $\hat{y}_{J}$ are crossing thresholds, and where all $\hat{y}_{i}$ solutions match their counterpart $y_{i}$ solutions within $\mathcal{R}$. 
Lemma 62 In the protein-only system (8), fix a black wall $w(x, x)$ and a region $\mathcal{R}=\mathcal{R}_{x} \times \mathcal{R}_{y_{J}} \times \prod_{i \neq J} \mathcal{R}_{y_{i}}$ as in Lemma 61. Let $\mathcal{W}$ be as in (22). Then, for $\epsilon$ sufficiently small, there exists a compact rectangular region $\hat{\mathcal{R}}(\epsilon)=$ $\hat{\mathcal{R}}_{x}(\epsilon) \times\left[\theta_{z}-\beta_{z}, \theta_{z}+\beta_{z}\right] \times \mathcal{R}_{y_{J}} \times \prod_{i \neq J} \mathcal{R}_{y_{i}}$ such that $\hat{\mathcal{R}}(\epsilon) \cap \mathcal{W} \neq \emptyset$, and if $\left(\hat{x}(0), \hat{y}_{i}(0), \hat{z}(0)\right) \in \hat{\mathcal{R}}(\epsilon)$, then

1. for $\hat{y}_{i}(0)=y_{i}(0), i=1, \ldots, n-1$, we have $\hat{y}_{i}(t)=y_{i}(t)$ as long as $y_{i}(t) \in$ $\mathcal{R}_{y_{i}}$

2. the forward trajectory $\left(\hat{x}(t), \hat{y}_{i}(t), \hat{z}(t)\right)$ remains in $\mathcal{R} \times\left[\theta_{z}-\beta_{z}, \theta_{z}+\beta_{z}\right]$ until the exit at $y_{J}=\hat{y}_{J}=\theta_{x, J}$.

Proof By the observation in Remark 41 the right hand side of equations for $\hat{y}_{i}$ and $y_{i}$ in (8) and (9) are identical. Since the projections of regions $\mathcal{R}$ and $\hat{\mathcal{R}}$ onto the $x$ and $\hat{x}$ variables, respectively, is identical, the inputs $X_{i}$ and $\hat{X}_{i}$ into the right hand side of the equations for $\hat{y}_{i}$ and $y_{i}$ are identical. This implies the first point. Therefore the exit hyperplanes $y_{J}=\hat{y}_{J}=\theta_{x, J}$ match. Moreover, $\left[\theta_{z}-\beta_{z}, \theta_{z}+\beta_{z}\right]$ is an invariant region for $\hat{z}$. So it only remains to decide the form of $\hat{\mathcal{R}}_{x}(\epsilon)$. By examination of the Poincaré map introduced in Section 6.3 below, there are oscillations in the $\hat{x}-\hat{z}$ feedback system about the point $\left(\theta_{x}, \theta_{z}\right)$. So the key to finding $\hat{\mathcal{R}}_{x}(\epsilon)$ is bounding the value of $\hat{x}$ into the interval $\mathcal{R}_{x}$, which we choose to write as $\mathcal{R}_{x}:=\left[\theta_{x}-\mu_{1}, \theta_{x}+\mu_{2}\right]$.

We claim that an interval of the form $\hat{\mathcal{R}}_{x}(\epsilon):=\left[\theta_{x}-\mu_{1}+\eta_{\epsilon}, \theta_{x}+\mu_{2}-\nu_{\epsilon}\right]$ is sufficient to prove the lemma, leading to a region

$\hat{\mathcal{R}}(\epsilon)=\hat{\mathcal{R}}_{x}(\epsilon):=\left[\theta_{x}-\mu_{1}+\eta_{\epsilon}, \theta_{x}+\mu_{2}-\nu_{\epsilon}\right] \times\left[\theta_{z}-\beta_{z}, \theta_{z}+\beta_{z}\right] \times \mathcal{R}_{y_{J}} \times \prod_{i \neq J} \mathcal{R}_{y_{i}}$

with adjustments

$$
\begin{aligned}
& \eta_{\epsilon}>\left(2^{\epsilon \gamma_{x}}-1\right)\left(\theta_{x}-\mu_{1}-\Phi_{x}(\bar{\kappa})\right) \\
& \nu_{\epsilon}>\left(2^{\epsilon \gamma_{x}}-1\right)\left(\Phi_{x}(\kappa)-\theta_{x}-\mu_{2}\right)
\end{aligned}
$$

for $\Phi_{x}(\bar{\kappa})<\theta_{x}$ and $\Phi_{x}(\kappa)>\theta_{x}$. Notice that these lower bounds shrink to 0 as $\epsilon \rightarrow 0$, so that $\hat{\mathcal{R}}_{x}(\epsilon) \rightarrow \mathcal{R}_{x}$. We remark that if $\Phi_{x}(\bar{\kappa}) \in\left[\theta_{x}-\mu_{1}, \theta_{x}\right)$ and $\Phi_{x}(\kappa) \in\left(\theta_{x}, \theta_{x}+\mu_{2}\right]$, then $\eta_{\epsilon}=\nu_{\epsilon}=0$. However, $\mathcal{R}_{x}$ may always be taken sufficiently small so that $\Phi_{x}(\bar{\kappa})<\theta_{x}-\mu_{1}$ and $\Phi_{x}(\kappa)>\theta_{x}+\mu_{2}$. We observe that $\hat{\mathcal{R}}(\epsilon) \cap \mathcal{W} \neq \emptyset$ by construction.

To prove the claim, consider taking the left endpoint of $\hat{\mathcal{R}}_{x}(\epsilon)$ as an initial condition, $x(0)=\theta_{x}-\mu_{1}+\eta_{\epsilon}$. If $\hat{z}(0) \in\left(\theta_{z}, \theta_{z}+\beta_{z}\right]$, then $\hat{x}$ is decreasing. We want to ensure that $\hat{x}$ will not decrease below $\theta_{x}-\mu_{1}$ in the time it takes $\hat{z}$ to reach $\theta_{z}$ and reverse the $\hat{x}$ flow. The time it takes $\hat{z}$ to reach $\theta_{z}$ from its extremal location $\theta_{z}+\beta_{z}$ is calculated from (11):

$$
\hat{T}_{z}=-\epsilon \ln \left(\frac{\theta_{z}-\left(\theta_{z}-\beta_{z}\right)}{\left(\theta_{z}+\beta_{z}\right)-\left(\theta_{z}-\beta_{z}\right)}\right)=\epsilon \ln 2 .
$$

Therefore, we require that the time taken for $\hat{x}$ to reach $\theta_{x}-\mu_{1}$ must not exceed $\epsilon \ln 2$. Again making use of (11), denoting $\Phi_{x}(\bar{\kappa})<\theta_{x}$ to be the focal 
point of $\hat{x}$ when $\hat{z}>\theta_{z}$, we find the travel time of $\hat{x}$ from $\theta_{x}-\mu_{1}+\eta_{\epsilon}$ to $\theta_{x}-\mu_{1}$ to be

$$
\hat{T}_{x}=\frac{1}{\gamma_{x}} \ln \left(1+\frac{\eta_{\epsilon}}{\theta_{x}-\mu_{1}-\Phi_{x}(\bar{\kappa})}\right) .
$$

Solving $\hat{T}_{x}<\epsilon \ln 2$ gives us the first expression in (24); a similar calculation yields the second expression for $\nu_{\epsilon}$.

Point (III) of Theorem 43 takes more work to establish. We will first reduce the protein-only (8) and PTM (9) systems into the study of two- and threedimensional systems respectively. We will then introduce a Poincaré map in the three-dimensional system to describe and quantify the oscillations in the PTM system as they are projected onto the $\hat{x}-\hat{z}$ plane. With the Poincaré map as a tool, we will then prove Points (III)a and (III)b of Theorem 43.

6.1 Simplification of the protein-only system near a black wall

We consider initial conditions $I^{0} \in \mathcal{R}$. For $\left(x\left(t ; I^{0}\right), y_{i}\left(t ; I^{0}\right)\right) \in \mathcal{R}$, the effects of $y_{i}$ for $i \in\{1, \ldots, J-1, J+1, \ldots, n-1\}$ on the right hand sides of $\left\{\dot{x}, \dot{y}_{i}\right\}_{i=1}^{n-1}$ are constant. Similarly, $x$ and $y_{J}$ have a constant effect on $\left\{\dot{y}_{i}\right\}_{i=1}^{n-1}$. In other words, the only non-constant values in (8) on the region $\mathcal{R}$ occur in the equation for $\dot{x}$, so that system (8) may be rewritten as

$$
\begin{aligned}
\dot{x} & =-\gamma_{x} x+e_{1} X^{ \pm}+e_{2} Y_{x, y_{J}}^{ \pm}+e_{3} X^{ \pm} Y_{x, y_{J}}^{ \pm}+C_{x} \\
\dot{y}_{J} & =-\gamma_{J} y_{J}+C_{J} \\
\dot{y}_{i} & =-\gamma_{i} y_{i}+C_{i}, \quad i \in\{1, \ldots, J-1, J+1, \ldots, n-1\} .
\end{aligned}
$$

The equation for $\dot{x}$ is a general multilinear form for a two-dimensional system. The constant $C_{x}$ depends on the locations of $y_{i}$ with respect to their thresholds. The constants $\left\{C_{i}\right\}_{i=1}^{n-1}$ depend on the locations $\left\{x, y_{i}\right\}_{i=1}^{n-1}$ with respect to their thresholds. The signs of $e_{i}$ and the superscripts \pm must be consistent with negative self-regulation in $x$.

Remark 63 The key observation that simplifies the analysis is that equations (25) and (26) are decoupled from (27) in the region $\mathcal{R}$. The solutions of (27) in $\mathcal{R}$ are exponentially decaying toward the focal points of the $y_{i}$ variables without crossing thresholds. Therefore, it is sufficient to study the two-dimensional system. Therefore, it is sufficient to study the local twodimensional system (25)-(26) near the black wall, even in the case where there are other self-regulating variables $y_{i}$.

By combining the terms and only considering the relevant threshold, the system is

$$
\begin{aligned}
& \dot{x}=-\gamma_{x} x+ \begin{cases}A, x>\theta_{x}, & y>\theta_{y} \\
B, x>\theta_{x}, & y<\theta_{y} \\
C, x<\theta_{x}, & y>\theta_{y} \\
D, x<\theta_{x}, & y<\theta_{y}\end{cases} \\
& \dot{y}=-\gamma_{y} y+E
\end{aligned}
$$


where we have written $y_{J}$ as $y$ and $\theta_{x, J}$ as $\theta_{y}$ for simpler notation. We will now also write $\mathcal{R}^{2}=\mathcal{R}_{x} \times \mathcal{R}_{y}$ to denote the region of initial conditions of interest in the two dimensional system. $\mathcal{R}^{2}$ can be thought of as the two lower cells in Figure 9 (see also Figure 12).

The hyperplanes $x=\theta_{x}$ and $y=\theta_{y}$ divide the two dimensional phase space into four cells. These cells will be named $\kappa_{A}-\kappa_{D}$ using the convention that in $\kappa_{U}$ the focal point of $x$ is $\Phi_{x}(U):=U / \gamma_{x}$ for $U \in\{A, B, C, D\}$, see Figure 9 (b). The system (28) has a black wall if $\Phi_{x}(A)<\theta_{x}<\Phi_{x}(C)$ or $\Phi_{x}(B)<\theta_{x}<$ $\Phi_{x}(D)$. In the remainder of the work, we assume without loss of generality that $\Phi_{x}(B)<\theta_{x}<\Phi_{x}(D)$ defines the black wall $w(x, x)$, see Figure $9(\mathrm{~b})$. The order of $\Phi_{x}(A), \Phi_{x}(C)$, and $\theta_{x}$ determines whether the adjoining wall is white or transparent, and whether or not $y$ is an up-regulator or down-regulator of $x$ in this region. To ensure an exit from this black wall in Figure 9 we have chosen that $\Phi_{x}(A), \Phi_{x}(C)<\theta_{x}$. Our choices are for illustration; our arguments work for all $A, B, C, D$ that can result from (25) - (27), provided that either $\Phi_{x}(A)<\theta_{x}<\Phi_{x}(C)$ or $\Phi_{x}(B)<\theta_{x}<\Phi_{x}(D)$.

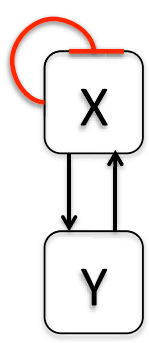

(a)

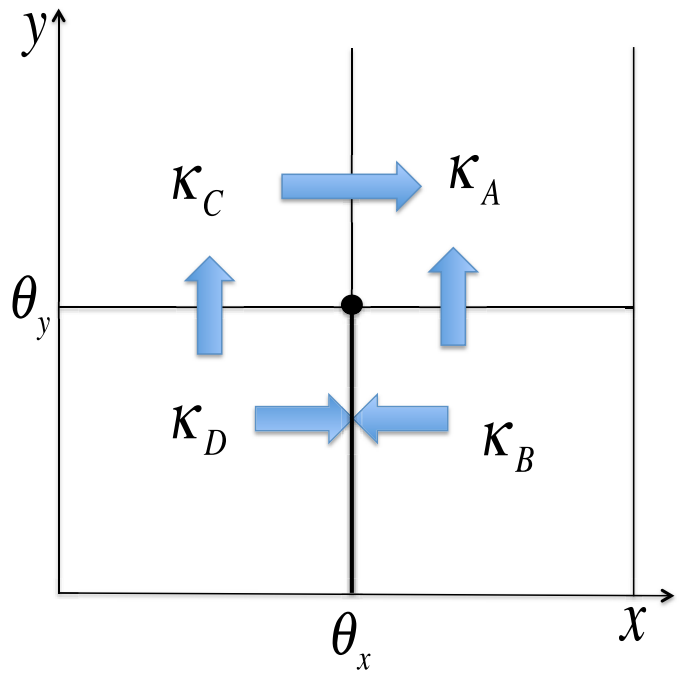

(b)

Fig. 9: (a) The reduced regulatory network for the protein-only system (8) assuming $x$ and $y$ mutually up-regulate and (b) a schematic of the dynamics near the black wall $w(x, x)$ for the parameter choice $\Phi_{x}(B)<\theta_{x}<\Phi_{x}(D)$ and $\Phi_{x}(A), \Phi_{x}(C)>\theta_{x}$. 
6.2 Simplification of PTM system near a feedback plane

We write the $n+1$ dimensional PTM system within $\hat{\mathcal{R}}(\epsilon)$ as:

$$
\begin{gathered}
\dot{\hat{x}}=-\gamma_{x} x+ \begin{cases}A, \hat{z}>\theta_{z}, & \hat{y}>\theta_{y} \\
B, \hat{z}>\theta_{z}, & \hat{y}<\theta_{y} \\
C, \hat{z}<\theta_{z}, & \hat{y}>\theta_{y} \\
D, \hat{z}<\theta_{z}, & y<\theta_{y}\end{cases} \\
\dot{\hat{z}}=\frac{1}{\epsilon}\left(-\hat{z}+\left\{\begin{array}{l}
\theta_{z}+\beta_{z}, \hat{x}>\theta_{x} \\
\theta_{z}-\beta_{z}, \hat{x}<\theta_{x}
\end{array}\right)\right. \\
\dot{\hat{y}}_{J}=-\gamma_{J} \hat{y}_{J}+E \\
\dot{\hat{y}}_{i}=-\gamma_{i} \hat{y}_{i}+C_{i}, i=1, \ldots, J-1, J+1, n-1
\end{gathered}
$$

All of the parameters match between the PTM and protein-only systems in $\hat{\mathcal{R}}(\epsilon)$ and $\mathcal{R}$ respectively. In particular, $E=C_{J}$ and $C_{i}$ in (29) match $C_{i}$, $i=1, \ldots, n-1$ in (27) due to Lemma 62 . The parameters $A-D$ in (28) and (29) are the same due to Remark 41 and because $\hat{z}$ down-regulates $\hat{x}$.

The equality in parameters means that the focal point components between systems are equal, $\hat{\Phi}_{x}=\Phi_{x}$ and $\hat{\Phi}_{y}=\Phi_{y}$, so we will omit the hats on the focal points. As in (28), we analyze the case where $\Phi_{x}(B)<\theta_{x}<\Phi_{x}(D)$. We study the decoupled three dimensional system

$$
\begin{aligned}
& \dot{\hat{x}}=-\gamma_{x} x+ \begin{cases}A, \hat{z}>\theta_{z}, & \hat{y}>\theta_{y} \\
B, \hat{z}>\theta_{z}, & \hat{y}<\theta_{y} \\
C, \hat{z}<\theta_{z}, & \hat{y}>\theta_{y} \\
D, \hat{z}<\theta_{z}, & y<\theta_{y}\end{cases} \\
& \dot{\hat{z}}=\frac{1}{\epsilon}\left(-\hat{z}+\left\{\begin{array}{l}
\theta_{z}+\beta_{z}, \hat{x}>\theta_{x} \\
\theta_{z}-\beta_{z}, \hat{x}<\theta_{x}
\end{array}\right)\right. \\
& \dot{\hat{y}}=-\gamma_{y} \hat{y}+E
\end{aligned}
$$

pictured in Figure 10, where we dropped index $J$ in $y$ equation. Then the $\hat{x}$ and $\hat{y}$ variables are directly comparable to $x$ and $y$ in (28). Analogously to $\mathcal{R}^{2}$, we denote by $\hat{\mathcal{R}}^{3}(\epsilon)$ the projection of $\hat{\mathcal{R}}(\epsilon)$ onto the $3 \mathrm{D}$-system. The three dimensional domain shown in Figure 10 with a schematic of the flow in $\hat{\mathcal{R}}^{3}(\epsilon)$.

As in (28), the cube $\hat{\mathcal{R}}^{3}(\epsilon)$ is divided into cells, $\kappa_{B,+} \times \mathcal{R}_{y}, \kappa_{B,-} \times \mathcal{R}_{y}$, $\kappa_{D,+} \times \mathcal{R}_{y}$, and $\kappa_{D,-} \times \mathcal{R}_{y}$. The projections of these cells onto the $(\hat{x}, \hat{z})$ plane are shown in Figure 11. The notation means the following: $\kappa_{U, \pm} \times \mathcal{R}_{y}$ has a focal point of $\left(\Phi_{x}(U), \Phi_{y}, \theta_{z} \pm \beta_{z}\right)$.

\subsection{Poincaré map}

In this section we will define a Poincare map for (30). Let

$$
\hat{S}_{1}:=\left\{(\hat{x}, \hat{y}, \hat{z}) \mid \hat{x} \geq \theta_{x}, \hat{z}=\theta_{z}\right\} \quad \text { and } \quad \hat{S}_{2}:=\left\{(\hat{x}, \hat{y}, \hat{z}) \mid \hat{x} \leq \theta_{x}, \hat{z}=\theta_{z}\right\} \text {. }
$$




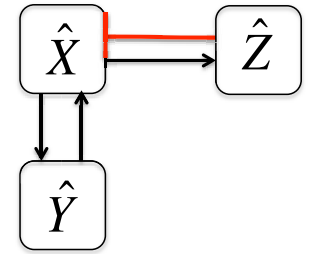

(a)

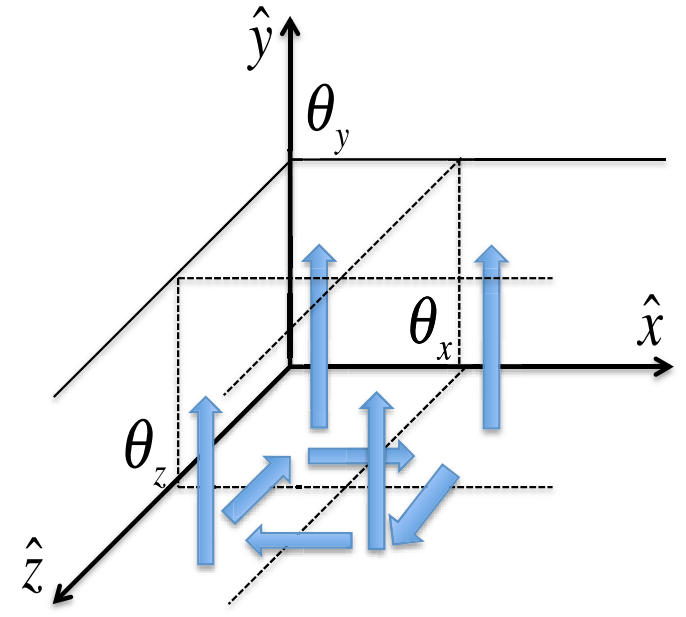

(b)

Fig. 10: (a) The regulation in (30) using mutual up-regulation for $\hat{x}$ and $\hat{y}$, as in the proteinonly system in Figure 9 , and (b) the local dynamics near $\hat{x}=\theta_{x}$ assuming $\hat{y}$ is increasing as in Figure 9. The cube with $\hat{y}$ increasing is the projection of $\hat{\mathcal{R}}(\epsilon)$ onto the 3D-system, $\hat{\mathcal{R}}^{3}(\epsilon) . \mathcal{W}$ from $(22)$ is the plane $\hat{x}=\theta_{x}$ slicing the cube down the center.

Let $\Psi(\hat{x}, \hat{y}, \hat{z}, t)$ be the flow generated by $(30)$ in $\hat{\mathcal{R}}^{3}(\epsilon) \backslash\left\{y=\theta_{y}\right\}$. Note that the equations for the $\hat{x}(t)$ and $\hat{z}(t)$ trajectories are decoupled from the $\hat{y}$ variable on $\hat{\mathcal{R}}^{3}(\epsilon) \backslash\left\{y=\theta_{y}\right\}$. Therefore the projection of $\Psi(\hat{x}, \hat{y}, \hat{z}, t)$ onto the first two variables $(\hat{x}, \hat{z})$

$$
\varphi(\hat{x}, \hat{z}, t):=(\hat{x}(t), \hat{z}(t))
$$

is a well defined flow and so $\Psi(\hat{x}, \hat{y}, \hat{z}, t)=(\varphi(\hat{x}, \hat{z}, t), y(t))$ is a product flow.

Therefore it is sufficient to study the flow $\varphi(\hat{x}, \hat{z}, t)$ in the $\hat{x}(t)-\hat{z}(t)$ plane. It is clear from the $\hat{x}-\hat{z}$ vector field pictured in Figure 11 that as long as $y$ does not cross $\theta_{y}$, the flow $\varphi(\hat{x}, \hat{z}, t)$ will alternately cross $\hat{x}=\theta_{x}$ and $\hat{z}=\theta_{z}$, thus oscillating in the $(\hat{x}, \hat{z})$ plane. Let

$$
S_{1}:=\left\{(\hat{x}, \hat{z}) \mid \hat{x} \geq \theta_{x}, \hat{z}=\theta_{z}\right\} \quad \text { and } \quad S_{2}:=\left\{(\hat{x}, \hat{z}) \mid \hat{x} \leq \theta_{x}, \hat{z}=\theta_{z}\right\}
$$

The interiors int $S_{1}$ and int $S_{2}$ are transverse sections of $\varphi$. The point $S_{1} \cap S_{2}=$ $\left(\theta_{x}, \theta_{z}\right)$ is an equilibrium of the flow $\varphi$, which we can observe from the closed form of the Poincaré map that we now introduce. Let $g_{1}: S_{1} \rightarrow S_{2}$ and $g_{2}: S_{2} \rightarrow S_{1}$ be $\varphi$-defined maps. The composition $P=g_{2} \circ g_{1}: S_{1} \rightarrow S_{1}$ defines a Poincaré map on $S_{1}$.

Let $\hat{I}^{0}=\left(\hat{x}(0), \theta_{z}\right)$ be an initial condition on $S_{1} \cap \hat{\mathcal{R}}^{3}(\epsilon)$. We find a closed form solution for $g_{1}: S_{1} \rightarrow S_{2}$ by calculating the time $\hat{T}_{x}$ needed to reach the line $x=\theta_{x}$ from $\hat{I}^{0}$, and then the time $\hat{T}_{z}$ needed to reach $S_{2}$ from $\left(\theta_{x}, \hat{z}\left(\hat{T}_{x}\right)\right)$. Then $g_{1}(\hat{x}(0)):=\varphi\left(\hat{T}_{z}+\hat{T}_{x}, \hat{x}(0)\right)$. The calculation makes repeated use of 


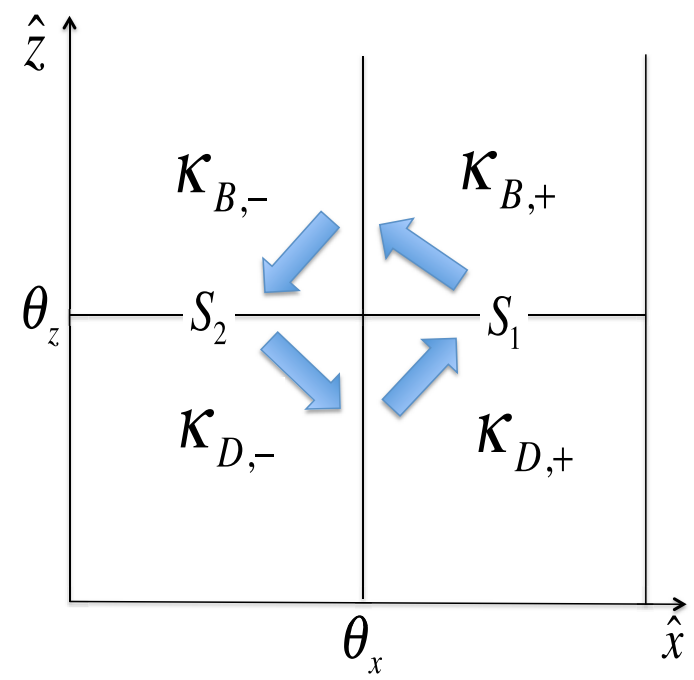

Fig. 11: A schematic of the flow in the $\hat{x}-\hat{z}$ system, showing the location of the Poincaré sections $S_{1}$ and $S_{2}$ and the quadrants where $\kappa_{U, \pm}$ means that the focal point in that quadrant is given by $\left(\Phi_{x}(U), \theta_{z} \pm \beta_{z}\right)$. These focal points and the fact that $\Phi_{x}(B)<\theta_{x}<\Phi_{x}(D)$ define the vector field.

(10)-(11)

$$
\begin{array}{ll}
\hat{T}_{x}^{1}=-\frac{1}{\gamma_{x}} \ln \left(\frac{\theta_{x}-\Phi_{x}(B)}{\hat{x}(0)-\Phi_{x}(B)}\right) ; \quad \hat{z}\left(\hat{T}_{x}^{1}\right)=\theta_{z}+\beta_{z}\left(1-e^{-\hat{T}_{x}^{1} / \epsilon}\right) \\
\hat{T}_{z}^{1}=-\epsilon \ln \left(\frac{\beta_{z}}{\hat{z}\left(\hat{T}_{x}^{1}\right)-\theta_{z}+\beta_{z}}\right) ; \quad \hat{x}\left(\hat{T}_{z}^{1}\right)=\Phi_{x}(B)+\left(\theta_{x}-\Phi_{x}(B)\right) e^{-\gamma_{x} \hat{T}_{z}^{1}}
\end{array}
$$

for $\hat{x}(0) \in S_{1}$, which yields

$$
g_{1}(\hat{x}(0))=\hat{x}\left(\hat{T}_{z}^{1}\right)=\Phi_{x}(B)+\left(\theta_{x}-\Phi_{x}(B)\right)\left(2-\left(\frac{\theta_{x}-\Phi_{x}(B)}{\hat{x}(0)-\Phi_{x}(B)}\right)^{1 / \epsilon \gamma_{x}}\right)^{-\epsilon \gamma_{x}}
$$

Similarly, the map $g_{2}: S_{2} \rightarrow S_{1}$, is given by calculating:

$$
\begin{aligned}
& \bar{T}_{x}^{1}=-\frac{1}{\gamma_{x}} \ln \left(\frac{\Phi_{x}(D)-\theta_{x}}{\Phi_{x}(D)-\hat{x}\left(\hat{T}_{z}^{1}\right)}\right) ; \quad \hat{z}\left(\bar{T}_{x}^{1}\right)=\theta_{z}-\beta_{z}\left(1-e^{-\bar{T}_{x}^{1} / \epsilon}\right) \\
& \bar{T}_{z}^{1}=-\epsilon \ln \left(\frac{\beta_{z}}{\theta_{z}+\beta_{z}-\hat{z}\left(\bar{T}_{x}^{1}\right)}\right) ; \quad \hat{x}\left(\bar{T}_{z}^{1}\right)=\Phi_{x}(D)+\left(\theta_{x}-\Phi_{x}(D)\right) e^{-\gamma_{x} \bar{T}_{z}^{1}}
\end{aligned}
$$


for $\hat{x}(0) \in S_{2}$, which yields

$$
g_{2}(\hat{x}(0))=\Phi_{x}(D)+\left(\theta_{x}-\Phi_{x}(D)\right)\left(2-\left(\frac{\Phi_{x}(D)-\theta_{x}}{\Phi_{x}(D)-\hat{x}\left(\hat{T}_{z}^{1}\right)}\right)^{1 / \epsilon \gamma_{x}}\right)^{-\epsilon \gamma_{x}}
$$

The first return time of the Poincaré map is then given by

$$
\begin{aligned}
T_{R}^{1}:= & \hat{T}_{x}^{1}+\hat{T}_{z}^{1}+\bar{T}_{x}^{1}+\bar{T}_{z}^{1} \\
= & -\frac{1}{\gamma_{x}} \ln \left(\frac{\left(\theta_{x}-\Phi_{x}(B)\right)\left(\Phi_{x}(D)-\theta_{x}\right)}{\left(\hat{x}(0)-\Phi_{x}(B)\right)\left(\Phi_{x}(D)-\hat{x}\left(\hat{T}_{z}^{1}\right)\right)}\right) \\
& -\epsilon \ln \left(\frac{\beta_{z}^{2}}{\left(\hat{z}\left(\hat{T}_{x}^{1}\right)-\theta_{z}+\beta_{z}\right)\left(\theta_{z}+\beta_{z}-\hat{z}\left(\bar{T}_{x}^{1}\right)\right)}\right)
\end{aligned}
$$

We observe that

$$
\lim _{\epsilon \rightarrow 0} T_{R}^{1}=\hat{T}_{x}^{1},
$$

since $\hat{x}\left(\hat{T}_{z}^{1}\right) \rightarrow \theta_{x}, \hat{z}\left(\hat{T}_{x}^{1}\right) \rightarrow \theta_{z}+\beta_{z}$, and $\hat{z}\left(\bar{T}_{x}^{1}\right) \rightarrow \theta_{z}-\beta_{z}$.

We now show that for more than one oscillation about the point $\left(\theta_{x}, \theta_{z}\right)$, the additional return time for each oscillation goes to zero as $\epsilon \rightarrow 0$. To see this, we first note that the expression (38) can be viewed as a function of four values that characterize four consecutive interceptions with the $x=\theta_{x}$ and $z=\theta_{z}$ thresholds

$$
T_{R}^{1}=F\left(\hat{x}(0), \hat{z}\left(\hat{T}_{x}^{1}\right), \hat{x}\left(\hat{T}_{z}^{1}\right), \hat{z}\left(\bar{T}_{x}^{1}\right)\right) .
$$

Then the return time for the $n$-th oscillation $T_{R}^{n}$ for $n>1$ can be written as

$$
T_{R}^{n}:=\hat{T}_{x}^{n}+\hat{T}_{z}^{n}+\bar{T}_{x}^{n}+\bar{T}_{z}^{n}=F\left(\hat{x}\left(\sum_{i=1}^{n-1} T_{R}^{i}\right), \hat{z}\left(\hat{T}_{x}^{n}\right), \hat{x}\left(\hat{T}_{z}^{n}\right), \hat{z}\left(\bar{T}_{x}^{n}\right)\right) .
$$

Lemma 64 For a fixed $n$, the $n$-th return time $\sum_{i=1}^{n} T_{R}^{i}$ can be written as

$$
\sum_{i=1}^{n} T_{R}^{i}=\hat{T}_{x}^{1}+\sum_{i=1}^{n} f(\epsilon ; i) \quad \text { with } \lim _{\epsilon \rightarrow 0} f(\epsilon ; i)=0 .
$$

Proof Given (39), it is sufficient to show that $T_{R}^{i}=\hat{T}_{x}^{i}+\hat{T}_{z}^{i}+\bar{T}_{x}^{i}+\bar{T}_{z}^{i}$ approaches zero as $\epsilon \rightarrow 0$ for all $2 \leq i \leq n$. We will make use of Equations (32)-(33) and (35)-(36) for a general $i$.

First we show that for any fixed $i$

$$
\lim _{\epsilon \rightarrow 0} \hat{T}_{z}^{i}+\bar{T}_{x}^{i}+\bar{T}_{z}^{i}=0 .
$$

To see this, we observe that by (33) and (36) both $\hat{T}_{z}^{i}$ and $\bar{T}_{z}^{i}$ are proportional to $\epsilon$, and so they tend to zero as $\epsilon \rightarrow 0$. Now consider the middle term $\bar{T}_{x}^{i}$ in (42) and its formula in (35). Note that this expression depends on the term 
$\hat{x}\left(\hat{T}_{z}^{i}\right)$, given by (33). By examining (33) and recalling that $\lim _{\epsilon \rightarrow 0} \hat{T}_{z}^{i}=0$, we see that $\lim _{\epsilon \rightarrow 0} \hat{x}\left(\hat{T}_{z}^{i}\right)=\theta_{x}$. Thus $\lim _{\epsilon \rightarrow 0} \bar{T}_{x}^{i}=0$, by inspection of (35). This finishes the proof of (42).

We now show by induction that

$$
\lim _{\epsilon \rightarrow 0} T_{R}^{i}=0 \quad \text { for all } \quad i=2, \ldots, n .
$$

For $n=2$, we notice that by (38) and (40) the value $\hat{T}_{x}^{2}$ depends on $\hat{x}\left(T_{R}^{1}\right)$. However, by (39) and the fact that $\hat{T}_{x}^{1}$ is the time needed to reach $\theta_{x}$ from $\hat{x}(0)$

$$
\lim _{\epsilon \rightarrow 0} \hat{x}\left(T_{R}^{1}\right)=\hat{x}\left(\hat{T}_{x}^{1}\right)=\theta_{x} .
$$

Therefore by (32) with $\hat{x}\left(T_{R}^{1}\right)$ replacing $\hat{x}(0)$ we conclude $\lim _{\epsilon \rightarrow 0} \hat{T}_{x}^{2}=0$. Finally, using (42), we have $\lim _{\epsilon \rightarrow 0} T_{R}^{2}=\hat{T}_{x}^{2}+\hat{T}_{z}^{2}+\bar{T}_{x}^{2}+\bar{T}_{z}^{2}=0$, which proves the induction step with $n=2$.

Proceeding to a general $n$, we make an inductive assumption that $\lim _{\epsilon \rightarrow 0} T_{R}^{i}=$ 0 for $i=2, \ldots, n-1$. We wish to show that $\lim _{\epsilon \rightarrow 0} T_{R}^{n}=0$. We first note that by the inductive assumption and (39) we have

$$
\lim _{\epsilon \rightarrow 0} \sum_{i=1}^{n-1} T_{R}^{i}=\hat{T}_{x}^{1}
$$

Then, since $\hat{T}_{x}^{1}$ is the time needed to reach $\theta_{x}$ it follows that

$$
\lim _{\epsilon \rightarrow 0} \hat{x}\left(\sum_{i=1}^{n-1} T_{R}^{i}\right)=\theta_{x}
$$

Using that fact plus (32) and (40), we have

$$
\lim _{\epsilon \rightarrow 0} \hat{T}_{x}^{n}=\frac{1}{\gamma_{x}} \ln \left(\frac{\hat{x}\left(\sum_{i=1}^{n-1} T_{R}^{i}\right)-\Phi_{x}(B)}{\theta_{x}-\Phi_{x}(B)}\right)=0,
$$

and it follows from (42) that $\lim _{\epsilon \rightarrow 0} T_{R}^{n}=\hat{T}_{x}^{n}+\hat{T}_{z}^{n}+\bar{T}_{x}^{n}+\bar{T}_{z}^{n}=0$ as desired.

Lemma 65 The Poincaré map $P=g_{2} \circ g_{1}: S_{1} \rightarrow S_{1}$ is a contraction map.

Proof The Poincaré map has a derivative $\left|P^{\prime}\right|=\left|g_{2}^{\prime}\left(g_{1}\right)\right|\left|g_{1}^{\prime}\right|<1$. We calculate

$$
\begin{aligned}
g_{i}^{\prime} & =\left(\frac{A_{i}^{1 / \epsilon \gamma_{x}}}{2-A_{i}^{1 / \epsilon \gamma_{x}}}\right)^{1+\epsilon \gamma_{x}}, i=1,2 \\
A_{1}(\hat{x}(0)) & =\frac{\theta_{x}-\Phi_{x}(B)}{\hat{x}(0)-\Phi_{x}(B)} \\
A_{2}\left(g_{1}(\hat{x}(0))\right) & =\frac{\theta_{x}-\Phi_{x}(D)}{g_{1}(\hat{x}(0))-\Phi_{x}(D)}
\end{aligned}
$$


Observe that $0<A_{1}, A_{2}<1$ since $\Phi_{x}(B)<\theta_{x}<\hat{x}(0) \in S_{1}$ and $g_{1}(\hat{x}(0))<$ $\theta_{x}<\Phi_{x}(D) \in S_{2}$. Then the quotient in parentheses is less than 1 , so that $\left|P^{\prime}\right|<1$. We conclude $P$ is a contraction map and observe that the oscillations in $(\hat{x}(t), \hat{z}(t))$ decay toward the equilibrium $\left(\theta_{x}, \theta_{z}\right)$ of $\varphi$.

The following corollary is immediate.

Corollary 66 Given $\delta>0$, then for any $\epsilon>0$ and $T<T_{y}$ where $T_{y}$ is the exit time from $\mathcal{R}^{2} \times\left[\theta_{z}-\beta_{z}, \theta_{z}+\beta_{z}\right]$,

$$
(\hat{x}(T), \hat{z}(T)) \in\left[\theta_{x}-\delta, \theta_{x}+\delta\right] \times\left\{\theta_{z}\right\} \subset S_{1} \cup S_{2}
$$

implies $\hat{x}(t) \in\left[\theta_{x}-\delta, \theta_{x}+\delta\right]$ for $t \in\left[T, T_{y}\right]$.

\subsection{Point (III)a of Theorem 43}

For the proof of Point (III)a, we first describe in detail the correspondence between the dynamics of the $x$ and $\hat{x}$ variables in (28) and (30). Even when we require that $x(0)=\hat{x}(0)$, the $x$ and $\hat{x}$ components of the focal points, $\Phi_{x}$ and $\hat{\Phi}_{x}$, in the two- and three-dimensional systems may be different, because the focal point will depend on the choice of $\hat{z}$ in the interval $\left[\theta_{z}-\beta_{z}, \theta_{z}+\beta_{z}\right]$.

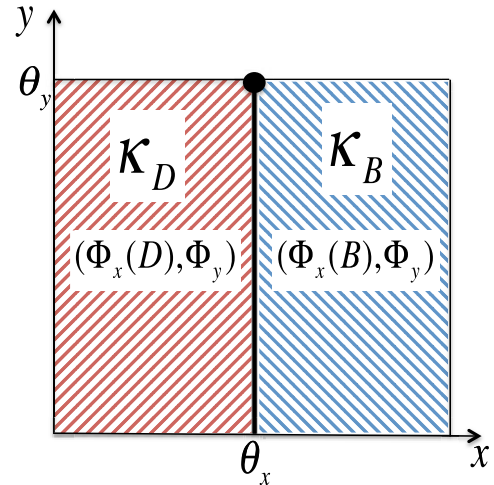

(a)

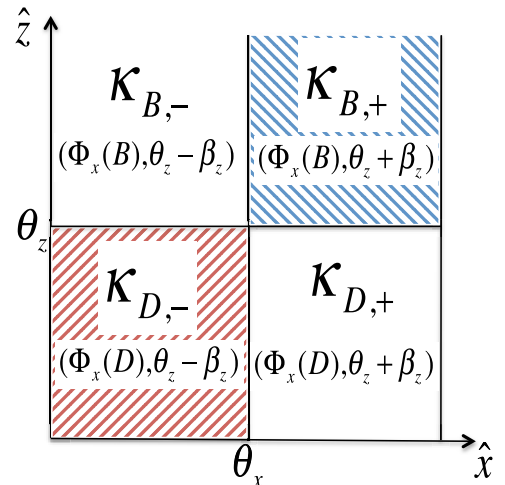

(b)

Fig. 12: The relationship between initial conditions $\mathcal{R}^{2}$ in the $x-y$ plane in (28) and those in $\hat{\mathcal{R}}^{3}(\epsilon)$ projected onto the $\hat{x}-\hat{z}$ plane in system (30). The colored regions in (a) and (b) correspond to matching directions of motion for $x$ and $\hat{x}$. In the red regions, $x$ and $\hat{x}$ are increasing. In the blue regions, they are decreasing. In the unshaded regions the $\hat{x}$ trajectory is headed away from the value $\theta_{x}$.

In Figure $12(\mathrm{a})$, we see the rectangular region $\mathcal{R}^{2}$ in the $(x, y)$ plane in system (28). In Figure 12 (b), depicting system (30), we see the projection of $\hat{\mathcal{R}}^{3}(\epsilon)$ onto the $(\hat{x}, \hat{z})$ plane. By inspection of $(28)$ and $(30)$, we see that $x$ and $\hat{x}$ share equations of motion in $\kappa_{B}$ and $\kappa_{B,+}$ with the $x$-component of the 
focal point $\Phi_{x}(B)$, and likewise in $\kappa_{D}$ and $\kappa_{D,-}$ with the $x$-component of the focal point $\Phi_{x}(D)$. In the other two quadrants, $\hat{x}$ is moving away from $\theta_{x}$, a phenomenon that never occurs in system (28). We say that $\kappa_{B,+} \cup \kappa_{D,-}$ is the matching region, and $\kappa_{B,-} \cup \kappa_{D,+}$ is the non-matching region.

Proof (Proof of Point (III)a of Theorem 43)

Recall that we wish to show that for $\delta>0$ and $\hat{I}^{0} \in \hat{\mathcal{R}}(\epsilon)$, there exists an $\epsilon\left(\delta, \hat{I}^{0}\right)$ such that $\left|\hat{x}\left(T_{y}\right)-x\left(T_{y}\right)\right| \leq \delta$. We shall assume for this proof that $x$ reaches the black wall in $(28)$, so that $x(t)=\theta_{x}$ for $t \in\left[T_{x}, T_{y}\right]$ for some $T_{x}<T_{y}$. A similar proof works for the case when $x$ does not reach the black wall, but we will not show the details here.

Corollary 66 states that if for some $T<T_{y}$

$$
\varphi(\hat{x}(0), \hat{z}(0), T) \in\left[\theta_{x}-\delta, \theta_{x}+\delta\right] \times\left\{\theta_{z}\right\} \subset S_{1} \cup S_{2},
$$

then $\hat{x}(t) \in\left[\theta_{x}-\delta, \theta_{x}+\delta\right]$ for $t \in\left[T, T_{y}\right]$. Therefore it is sufficient to find $\epsilon$ sufficiently small to ensure that $\hat{x}(T) \in\left[\theta_{x}-\delta, \theta_{x}+\delta\right] \times\left\{\theta_{z}\right\}$ for some $T<T_{y}$.

We will do this by splitting the trajectory $\varphi(\hat{x}(0), \hat{z}(0), t)$ into pieces that cross the matching and non-matching regions in an alternating fashion. The time taken to cross the matching regions is controlled by the decay rate $\gamma_{x}$ and cannot be altered, but the time taken to cross a non-matching region is bounded above by $\epsilon \ln 2$. To see this we set $\hat{z}(0)$ to its maximum and minimum possible values $\hat{z}(0)=\theta_{z} \pm \beta_{z}$ in Equation (11) for $\hat{T}_{z}$, the time taken to travel from $\hat{z}(0)$ to $\theta_{z}$ :

$$
\hat{T}_{z}=-\epsilon \ln \left(\frac{\theta_{z}-\left(\theta_{z} \mp \beta_{z}\right)}{\hat{z}(0)-\left(\theta_{z} \mp \beta_{z}\right)}\right) \leq \epsilon \ln 2
$$

Therefore our strategy will be to limit the time spent in the non-matching region by taking $\epsilon$ small.

We first consider $(\hat{x}(0), \hat{z}(0))$ in a matching region, shown in Figure 13 (a) for the example $(\hat{x}(0), \hat{z}(0)) \in \kappa_{B,+}$. A similar picture exists for $(\hat{x}(0), \hat{z}(0)) \in$ $\kappa_{D,-}$, and the following argument holds for both cells. The solutions $\hat{x}(t)$ and $x(t)$ are identical until they reach threshold $\theta_{x}$ after time $T_{x}$. At this point the solution $\varphi(\hat{x}(0), \hat{z}(0), t)$ enters the non-matching region while $x(t)=\theta_{x}$. The difference $|\hat{x}(t)-x(t)|$ will therefore grow for some time $t_{1}$ until $\varphi(\hat{x}(0), \hat{z}(0), t)$ reaches the next threshold $\hat{z}\left(T_{x}+t_{1}\right)=\theta_{z}$. We denote $\hat{x}_{1}:=\hat{x}\left(T_{x}+t_{1}\right)$. We want to chose $\epsilon$ small enough so that $t_{1}+T_{x}<T_{y}$ and $\left|\hat{x}_{1}-\theta_{x}\right| \leq \delta$.

Using (43) we choose $\epsilon_{1}>0$ such that $t_{1} \leq \epsilon_{1} \ln 2<T_{y}-T_{x}$. Then, noticing that

$$
\left|\hat{x}_{1}-\theta_{x}\right|=\left|\Phi_{x}+\left(\theta_{x}-\Phi_{x}\right) e^{-\gamma_{x} t_{1}}-\theta_{x}\right|,
$$

we choose $\epsilon_{2}>0$ such that

$$
t_{1} \leq \epsilon_{2} \ln 2 \leq-\frac{1}{\gamma_{x}} \ln \left(1-\frac{\delta}{\left|\Phi_{x}-\theta_{x}\right|}\right)
$$

This completes the matching case if we take $\epsilon \leq \min \left\{\epsilon_{1}, \epsilon_{2}\right\}$. 


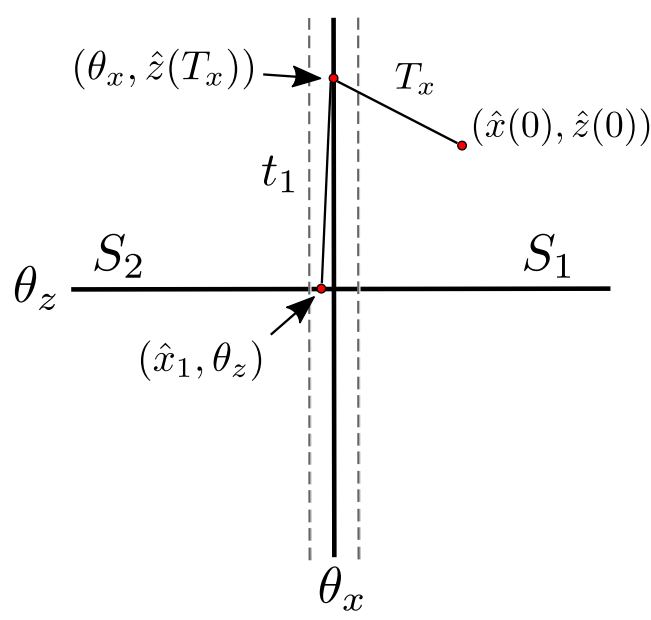

(a)

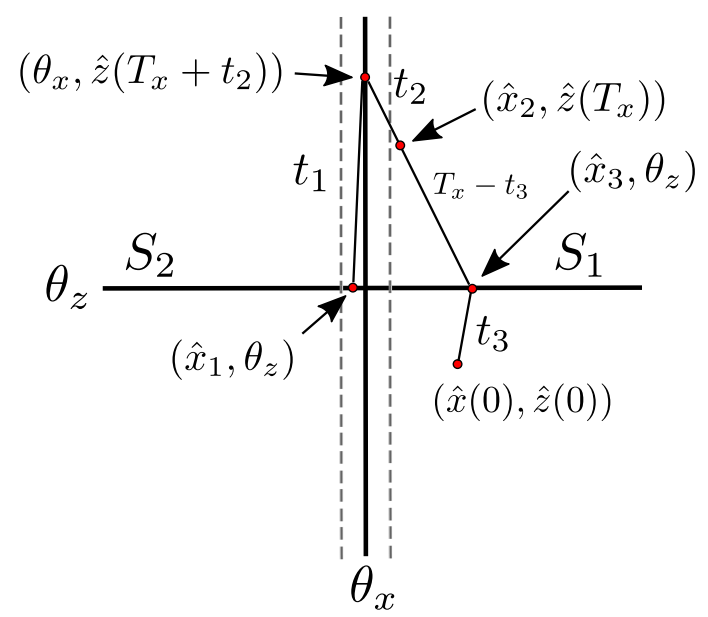

(b)

Fig. 13: Schematic demonstrating the proof of Point (III)a of Theorem 43. In reality, the straight line segments would be curves. Intermediate labels are given to points on the trajectory, and each intervening segment is labeled by the travel time along the segment. The dotted lines denote the region $\left[\theta_{x}-\delta, \theta_{x}+\delta\right] \times\left\{\theta_{z}\right\}$. (a) The initial condition is in a matching cell. After time $T_{x}$, the trajectory reaches the hyperplane $\hat{x}=\theta_{x}$. After a further time $t_{1}$, it intersects the hyperplane $\hat{z}=\theta_{z}$ within the $\delta$ region. (b) The initial condition is in a nonmatching cell. At time $T_{x}$, the trajectory has not yet reached $\hat{x}=\theta_{x}$; it requires a further time $t_{2}$ to arrive. Then the time $t_{1}$ to reach $\hat{z}=\theta_{z}$ is analogous to that in the matching case (a).

Now take $(\hat{x}(0), \hat{z}(0))$ in the non-matching region, as shown in Figure 13 (b) for the example $(\hat{x}(0), \hat{z}(0)) \in \kappa_{D,+}$. As before, the following argument also holds for $\kappa_{B,-}$. In the non-matching case, $x(0)$ has the $x$-component of the focal point $\Phi_{x}$ and $\hat{x}(0)$ has the $x$-component of the focal point $\Phi_{x}^{\prime}$. This means that if $(\hat{x}(0), \hat{z}(0)) \in \kappa_{B,-}$ then $\Phi_{x}=\Phi_{x}(B), \Phi_{x}^{\prime}=\Phi_{x}(D)$ while if $(\hat{x}(0), \hat{z}(0)) \in \kappa_{D,+}$ we have $\Phi_{x}=\Phi_{x}(D)$ and $\Phi_{x}^{\prime}=\Phi_{x}(B)$. We will need to analyze the crossing of three consecutive cells, while still applying the key estimate (45). We will step through the trajectory cell by cell, counting down a sequence of turning points $\hat{x}(0) \rightarrow \hat{x}_{3} \rightarrow \hat{x}_{2} \rightarrow \hat{x}_{1}$ in order to match the notation in the first case. The matching notation can be seen side-by-side in Figure 13.

We outline the gist of our argument here, with formulas to follow. Let $t_{3}$ be the time until $\varphi(\hat{x}(0), \hat{z}(0), t)$ reaches the $\theta_{z}$ threshold. If the initial condition satisfies $\left|\hat{x}(0)-\theta_{x}\right|=\left|x(0)-\theta_{x}\right| \geq \delta$, then $\hat{x}_{3}:=\hat{x}\left(t_{3}\right) \notin\left[\theta_{x}-\delta, \theta_{x}+\delta\right] \times\left\{\theta_{z}\right\}$. 
Therefore we must wait until the second encounter with $\theta_{z}$ threshold to ensure that $\hat{x}$ is in the desired region $\left[\theta_{x}-\delta, \theta_{x}+\delta\right] \times\left\{\theta_{z}\right\}$.

From $\left(\hat{x}_{3}, \theta_{z}\right)$, the trajectory $\varphi(\hat{x}(0), \hat{z}(0), t)$ enters a matching region. To compare this trajectory to the trajectory of the two dimensional system, we note that at time $T_{x}, x\left(T_{x}\right)=\theta_{x}$. However, $\varphi\left(\hat{x}(0), \hat{z}(0), T_{x}\right)$ has not finished crossing the matching region to reach $\theta_{x}$, because $\left|\hat{x}_{3}-\theta_{x}\right|>\left|\hat{x}(0)-\theta_{x}\right|$. Therefore $\hat{x}_{2}:=\hat{x}\left(T_{x}\right) \neq \theta_{x}$. We define $t_{2}$ to be the time it takes the trajectory to reach $\theta_{x}$; that is

$$
\varphi\left(\hat{x}(0), \hat{z}(0), T_{x}+t_{2}\right)=\left(\theta_{x}, \hat{z}_{2}\right) .
$$

At this point, $\hat{x}\left(T_{x}+t_{2}\right)=\theta_{x}$. Lastly we define a time $t_{1}$, which is the travel time from $\left(\theta_{x}, \hat{z}_{2}\right)$ to a point $\left(\hat{x}_{1}, \theta_{z}\right)$ where $\hat{x}_{1}:=\hat{x}\left(T_{x}+t_{2}+t_{1}\right)$. Analogously to the matching case, we must ensure that $\left|\hat{x}_{1}-\theta_{x}\right| \leq \delta$ and $T_{x}+t_{2}+t_{1}<T_{y}$.

The value of $\hat{x}(t)$ on its first visit to $\theta_{z}$ threshold is given by

$$
\hat{x}_{3}:=\Phi_{x}^{\prime}+\left(\hat{x}(0)-\Phi_{x}^{\prime}\right) e^{-\gamma_{x} t_{3}},
$$

and the value of $\hat{x}_{2}:=\hat{x}\left(T_{x}\right)$ is given by

$$
\hat{x}_{2}:=\Phi_{x}+\left(\hat{x}_{3}-\Phi_{x}\right) e^{-\gamma_{x}\left(T_{x}-t_{3}\right)} \neq \theta_{x} .
$$

The travel time from $\hat{x}_{2}$ to the threshold $\theta_{x}$ is given by the time $t_{2}$ :

$$
t_{2}=-\frac{1}{\gamma_{x}} \ln \left(\frac{\theta_{x}-\Phi_{x}}{\hat{x}_{2}-\Phi_{x}}\right) .
$$

Since by (43) we have $t_{3} \leq \epsilon \ln 2$, it is easy to see that $\hat{x}_{2} \rightarrow \theta_{x}$ as $\epsilon \rightarrow 0$, which implies $t_{2} \rightarrow 0$ as $\epsilon \rightarrow 0$.

To complete the argument, we need to estimate $t_{1}$. To do this we note that $\hat{x}\left(T_{x}+t_{2}\right)=\theta_{x}$ which puts us in the same situation that has been discussed in the matching region case. Therefore, we can use the formula as in (44) for $\left|\hat{x}_{1}-\theta_{x}\right|$ to estimate $t_{1} \leq \epsilon \ln 2$ as in (45). Thus we choose $\epsilon_{1}>0$ such that $t_{2}+t_{1}<T_{y}-T_{x}$, chose $\epsilon_{2}>0$ such that (45) holds, and then take $\epsilon<\min \left\{\epsilon_{1}, \epsilon_{2}\right\}$. This completes the proof.

\subsection{Point (III)b of Theorem 43}

The proof of Point (III)b requires some preliminary discussion, definitions, and lemmas. The excluded region $\mathcal{E}(\epsilon, \delta)$, is composed of initial conditions $\hat{I}^{0}$ that lead to trajectories in system (30) whose $\hat{x}$ component is insufficiently close to the corresponding $x$ trajectories in system (28). The symbolic description of $\mathcal{E}(\epsilon, \delta)$ in terms of inequalities and set operations is possible, but tedious. For our purposes, it is sufficient to describe some of the more interesting regions of $\tilde{\mathcal{E}}(\epsilon, \delta)$ and prove that they have non-empty interior, where recall that $\mathcal{E}(\epsilon, \delta)=$ $\tilde{\mathcal{E}}(\epsilon, \delta) \times\left(\prod_{i \neq J} \mathcal{R}_{y_{i}}\right)$, and $\tilde{\mathcal{E}}(\epsilon, \delta) \subset \hat{\mathcal{R}}^{3}(\epsilon)$.

We shall consider initial conditions $I^{0} \in \mathcal{R}^{2}$ where the time for the solution to reach the black wall $T_{x}$ is less than $T_{y}$, so that the trajectory starting at 
$I^{0}$ enters the black wall. We know that there is an open set of initial conditions in $\mathcal{R}^{2}$ satisfying this condition, so by the proof of Lemma 62 , there is a corresponding open set of initial conditions $\mathcal{I} \subset \hat{\mathcal{R}}^{3}(\epsilon)$ intersecting $\mathcal{W}$ from (22). Part of the excluded region $\tilde{\mathcal{E}}(\epsilon, \delta)$ is defined by the inequality $\left\{\hat{I}^{0} \in \mathcal{I}|| \hat{x}\left(T_{y} ; \hat{I}^{0}\right)-\theta_{x} \mid>\delta\right\}$. This is still a complicated set to describe exactly, so we will intersect it with the Poincaré section $S_{1}$. This projection of $\tilde{\mathcal{E}}(\epsilon, \delta)$ exhibits interesting "stripes" in the $(\hat{x}, \hat{z})$ phase space.

Definition 67 We denote the flow-defined map $h_{T_{y}}: \hat{\mathcal{R}}^{3}(\epsilon) \rightarrow\left\{\hat{y}=\theta_{y}\right\}$ via (30) as

$$
h_{T_{y}}:(\hat{x}(0), \hat{y}(0), \hat{z}(0)) \mapsto\left(\hat{x}\left(T_{y}\right), \theta_{y}, \hat{z}\left(T_{y}\right)\right) .
$$

This map takes the initial value $\hat{I}^{0}$ to its value at the time of exit $T_{y}$ from $\hat{\mathcal{R}}^{3}(\epsilon)$.

Our basic approach will be to take the preimage of the $\delta$-strip

$$
U(\delta):=\left[\theta_{x}-\delta, \theta_{x}+\delta\right] \times\left[\theta_{z}-\beta_{z}, \theta_{z}+\beta_{z}\right] \times\left\{y=\theta_{y}\right\}
$$

under $h_{T_{y}}$. The regions which fall outside of the preimage will not map into the desirable region $U(\delta)$. To motivate our work, we provide an illustration of the preimage of $U(\delta)$ projected onto $(\hat{x}, \hat{z})$ space in Figure 3 for increasing exit times $T_{y}$. The preimage really exists on some plane $\left\{y(0) \quad \mid y\left(T_{y}\right)=\right.$ $\theta_{y}$ for a fixed $\left.T_{y}\right\}$, so that the unions of the preimages over $T_{y}$ are stacked images like those in Figure 3, with the more twisted regions farther from the hyperplane $\left\{y=\theta_{y}\right\}$.

Definition 68 For $\delta>0$, let $P\left(\delta, T_{y}\right)=h_{T_{y}}^{-1}(U(\delta))$ be the preimage of the compact region $U(\delta)$ from (46). The set $P\left(\delta, T_{y}\right) \cap S_{1}$ is the collection of intervals

$$
P_{i}\left(\delta, T_{y}\right):=\left[\hat{x}_{i}^{*}-\alpha_{i}^{-}, \hat{x}_{i}^{*}+\alpha_{i}^{+}\right]
$$

where $h_{T_{y}}\left(\hat{x}_{i}^{*}, \theta_{z}, y(0)\right)=\left(\theta_{x}, z\left(T_{y}\right), \theta_{y}\right)$ and $h_{T_{y}}\left(\alpha_{i}^{+}, \theta_{z}, y(0)\right)=\left(\theta_{x}+\delta, \hat{z}\left(T_{y}\right), \theta_{y}\right)$. Due to the intersection with $S_{1}$, either $h_{T_{y}}\left(\left(\alpha_{i}^{-}, \theta_{z}, y(0)\right)=\left(\theta_{x}-\delta, \hat{z}\left(T_{y}\right), \theta_{y}\right)\right.$ or $\alpha_{i}^{-}=\hat{x}_{i}^{*}-\theta_{x}$ if the preimage value of $\theta_{x}-\delta$ is less than $\theta_{x}$.

The intervals $P_{i}$ are seen in Figure 3 as the intersection of the shaded regions with the horizontal axis to the right of $\hat{x}=\theta_{x}$. The first panel has one such interval, the second and third have two, and the fourth has three.

Observe that the region

$$
\bar{S}_{x}\left(\delta, T_{y}\right)=S_{1} \backslash \bigcup_{i} P_{i}\left(\delta, T_{y}\right)
$$

contains initial conditions that lead to solutions that will be outside of the $\delta$-strip in $\mathcal{R}^{3}(\epsilon)$ after time $T_{y}$. The set $\bar{S}_{x}\left(\delta, T_{y}\right) \cap\left\{\hat{x}(0) \mid T_{x}<T_{y}\right\}$ consists of initial data $\left(x(0), \theta_{z}, y(0)\right)$ such that their projection $(x(0), y(0))$ will reach the black wall in system (28), and subsequently slide along the black wall to 
the exit at $\left(\theta_{x}, \theta_{y}\right)$. Yet, solutions of system (30) starting in $\bar{S}_{x}\left(\delta, T_{y}\right) \cap\{\hat{x}(0) \mid$ $\left.T_{x}<T_{y}\right\}$ will exit $\mathcal{R}^{3}(\epsilon)$ outside of the $\delta$-strip surrounding $\left(\theta_{x}, \theta_{y}\right)$. If we define

$$
Q\left(\delta, T_{y}\right):=\left(\bar{S}_{x}\left(\delta, T_{y}\right) \cap\left\{\hat{x}(0) \mid T_{x}<T_{y}\right\}\right) \times\left\{\hat{z}=\theta_{z}\right\} \times\left\{y(0) \mid y\left(T_{y}\right)=\theta_{y}\right\},
$$

then

$$
\left(\hat{\mathcal{R}}^{3}(\epsilon) \cap \bigcup_{T_{y}} Q\left(\delta, T_{y}\right)\right) \subset \tilde{\mathcal{E}}(\epsilon, \delta) .
$$

To complete the proof of Theorem 43, it remains to show that the set in (48) is nonempty and can be widened to a region with nonempty interior. Observe that if $T_{y}^{1} \neq T_{y}^{2}$, then $Q\left(\delta, T_{y}^{1}\right) \cap Q\left(\delta, T_{y}^{2}\right)=\emptyset$, as can be seen in (47). Therefore (48) is a disjoint union parameterized by $T_{y}$ and it is sufficient for nonemptiness to show that $\hat{\mathcal{R}}^{3}(\epsilon) \cap Q\left(\delta, T_{y}\right) \neq \emptyset$ for one $T_{y}$. Several intermediate results will be of use.

Lemma 69 Consider an initial condition $\left(x(0), y(0), \theta_{z}\right) \in S_{1}$ and let $T_{y}$ be the exit time for the resulting solution of (30) from $\hat{\mathcal{R}}^{3}(\epsilon)$. Then $T_{y} \rightarrow 0$ if, and only if, $y(0) \rightarrow \theta_{y}$.

Proof By (11),

$$
T_{y}=-\frac{1}{\gamma_{y}} \ln \left(\frac{\theta_{y}-\Phi_{y}}{y(0)-\Phi_{y}}\right) .
$$

Notice that $y(0)$ uniquely defines the exit time $T_{y}$, and that $T_{y}$ approaches 0 only if $y(0) \rightarrow \theta_{y}$.

\section{Lemma 610 Some consequences of Definition 68.}

1. For a fixed $y(0)$ and associated $T_{y}$, there exists a maximal element $\hat{x}_{0}^{*}$ of the set $\left\{\hat{x}_{i}^{*}\right\}$ as given in Definition 68.

2. For a fixed $y(0)$ and associated $T_{y}$, if $\hat{x}(0) \in\left[\theta_{x}, \hat{x}_{0}^{*}\right)$ then $\hat{T}_{x}<T_{y}$ is satisfied, where $\hat{T}_{x}$ is the travel time to $\theta_{x}$ from (11)

$$
\hat{T}_{x}=-\frac{1}{\gamma_{x}} \ln \left(\frac{\theta_{x}-\Phi_{x}}{\hat{x}(0)-\Phi_{x}}\right) .
$$

3. For a fixed $y(0)$ and associated $T_{y}$, as $\delta \rightarrow 0$, the points $\alpha_{i}^{ \pm}$as given in Definition 68 satisfy $\alpha_{i}^{ \pm} \rightarrow \hat{x}_{i}^{*}$.

4. As $y(0) \rightarrow \theta_{y}$ and $T_{y} \rightarrow 0$, $\hat{x}_{0}^{*} \rightarrow \theta_{x}$.

Proof Fix $y(0)$ and therefore $T_{y}$. Define $\left(\hat{x}_{0}^{*}, \theta_{z}, y(0)\right) \in S_{1}$ to be the initial condition whose trajectory arrives at intersection of $\hat{x}=\theta_{x}$ and $y=\theta_{y}$ exactly at time $T_{y}$. The formula for $x_{0}^{*}$ is

$$
\hat{x}_{0}^{*}=\Phi_{x}(B)+\left(\theta_{x}-\Phi_{x}(B)\right) e^{\gamma_{x} T_{y}}
$$

by (10) and the fact that trajectories starting in $S_{1}$ flow through $\kappa_{B,+}$ as shown in Figure 11. 
By Definition 68, each $x_{i}^{*}$ is the preimage of $\theta_{x}$. Consider $\hat{x}(0)>\hat{x}_{0}^{*}$. By the monotonicity of each component of the flow in $\kappa_{B,+}$, the $x$ component of $h_{T_{y}}\left(\hat{x}(0), \theta_{z}, y(0)\right)$ is greater than the $\hat{x}$ component of $h_{T_{y}}\left(\hat{x}_{0}^{*}, \theta_{z}, y(0)\right)$, which is $\theta_{x}$. In other words, $\hat{x}\left(T_{y}\right)>\theta_{x}$ means $\hat{x}(0)$ cannot equal any $x_{i}^{*}$. Therefore $x_{0}^{*}$ is maximal, as desired in (1).

Now consider $\hat{x}(0)<x_{0}^{*}$. Again by monotonicity in $\kappa_{B,+}$, there exists a time $\hat{T}_{x}<T_{y}$ such that $h_{\hat{T}_{x}}\left(\hat{x}(0), \theta_{z}, y(0)\right)=\left(\theta_{x}, z\left(\hat{T}_{x}\right), y\left(\hat{T}_{x}\right)\right)$. This proves (2).

As $\delta \rightarrow 0$, by continuity we have that $\left(\hat{\alpha}_{i}^{ \pm}, \theta_{z}, y(0)\right)=h_{T_{y}}^{-1}\left(\theta_{x} \pm \delta, \hat{z}\left(T_{y}\right), \theta_{y}\right) \rightarrow$ $h_{T_{y}}^{-1}\left(\theta_{x}, \hat{z}\left(T_{y}\right), \theta_{y}\right)=\left(\hat{x}_{i}^{*}, \theta_{z}, y(0)\right)$. This proves $(3)$.

Now let $y(0) \rightarrow \theta_{y}$ and $T_{y} \rightarrow 0$. By the equation for $\hat{x}_{0}^{*}$ in (49), we see that $\hat{x}_{0}^{*} \rightarrow \theta_{x}$, as desired in (4).

Lemma 611 For sufficiently small $T_{y}$, there exists $\Delta(\epsilon)>0$ such that for $0<\delta<\Delta(\epsilon), Q\left(\delta, T_{y}\right) \cap \hat{\mathcal{R}}^{3}(\epsilon) \neq \emptyset$.

Proof We can choose $T_{y}$ sufficiently small so that $\hat{x}_{0}^{*}<\theta_{x}+\mu_{2}-\nu_{\epsilon}$ by Lemma 610 (4), and also small enough so that $y(0) \in \mathcal{R}_{y}$ by Lemma 69. Then $\left(x_{0}^{*}, y(0), \theta_{z}\right) \in \hat{\mathcal{R}}^{3}(\epsilon)$ as in $(23)$. Now let $\hat{x}_{1}^{*}$ be the next largest preimage of $\theta_{x}$ under $h_{T_{y}}$, so that $x_{i}^{*}<x_{1}^{*}<x_{0}^{*}$ for all $i$ using Lemma 610 (1). Then choose $\Delta(\epsilon)$ sufficiently small so that

1. $\hat{x}_{1}^{*}>\theta_{x}+\Delta(\epsilon)$, and

2. $\alpha_{1}^{+}<\alpha_{0}^{-}$.

The first can be accomplished by taking $\Delta(\epsilon)<\hat{x}_{1}^{*}-\theta_{x}$, and the second can be satisfied because $\alpha_{1}^{+} \rightarrow \hat{x}_{1}^{*}$ and $\alpha_{0}^{-} \rightarrow \hat{x}_{0}^{*}$ as $\Delta(\epsilon) \rightarrow 0$ by Lemma 610 (3).

By construction, the interval $\left(\alpha_{1}^{+}, \alpha_{0}^{-}\right) \subset \hat{\mathcal{R}}_{x}(\epsilon)$ is nonempty, maps to the right of $\left[\theta_{x}, \theta_{x}+\Delta(\epsilon)\right]$, and satisfies $\hat{T}_{x}<T_{y}$ by Lemma $610(2)$. Then the region

$$
Q\left(\Delta(\epsilon), T_{y}\right)=\left(\alpha_{1}^{+}, \alpha_{0}^{-}\right) \times\left\{\hat{z}=\theta_{z}\right\} \times\left\{y(0) \in \mathcal{R}_{y} \mid y\left(T_{y}\right)=\theta_{y}\right\} \subset \hat{\mathcal{R}}^{3}(\epsilon)
$$

is nonempty.

Now take $0<\delta<\Delta(\epsilon)$ while holding $T_{y}$ constant. We know that the preimages of the $\delta$ - and $\Delta(\epsilon)$-strips satisfy $P\left(\delta, T_{y}\right) \subset P\left(\Delta(\epsilon), T_{y}\right)$, so that

$$
\left(Q\left(\delta, T_{y}\right) \cap \hat{\mathcal{R}}^{3}(\epsilon)\right) \supset\left(Q\left(\Delta(\epsilon), T_{y}\right) \cap \hat{\mathcal{R}}^{3}(\epsilon)\right)
$$

is nonempty as desired.

To finish the proof of point (III)b of Theorem 43, choose a $T_{y}^{*}$ satisfying the requirements of Lemma 611 and fix the resulting $\Delta(\epsilon)$. For $0<\delta<\Delta(\epsilon)$, the excluded region

$$
\tilde{\mathcal{E}}(\epsilon, \delta) \supset\left(\hat{\mathcal{R}}^{3}(\epsilon) \cap \bigcup_{T_{y}} Q\left(\delta, T_{y}\right)\right) \supset\left(\hat{\mathcal{R}}^{3}(\epsilon) \cap Q\left(\delta, T_{y}^{*}\right)\right) \neq \emptyset .
$$


Therefore $\mathcal{E}(\epsilon, \delta)=\tilde{\mathcal{E}}(\epsilon, \delta) \times \prod_{i \neq J} \mathcal{R}_{y_{i}}$ is nonempty. Finally, continuous dependence on initial conditions assures that there is an open neighborhood around any initial condition in $Q\left(\delta, T_{y}^{*}\right)$ that also belongs to $\tilde{\mathcal{E}}(\epsilon, \delta)$. In other words, we can thicken the set in $y$ and $\hat{z}$, leading to a nonempty interior in $\hat{\mathcal{R}}^{3}(\epsilon)$. This completes the proof. 